\title{
Accelerating Families of Fuzzy K-Means Algorithms for Vector Quantization Codebook Design
}

\author{
Edson Mata ${ }^{1}$, Silvio Bandeira ${ }^{1}$, Paulo de Mattos Neto ${ }^{2}$, Waslon Lopes ${ }^{3, *}$ and Francisco Madeiro ${ }^{1}$ \\ 1 Center of Science and Technology, Catholic University of Pernambuco (UNICAP), Recife 50050-900, Brazil; \\ edsonmata@hotmail.com (E.M.); silvio@c3.unicap.br (S.B.); madeiro@c3.unicap.br (F.M.) \\ 2 Centro de Informática, Universidade Federal de Pernambuco (UFPE), Recife 50740-560, Brazil; \\ psgmn@cin.ufpe.br \\ 3 Department of Electrical Engineering, Center of Alternative and Renewable Energy, \\ Federal University of Paraíba (UFPB), João Pessoa 58038-130, Brazil \\ * Correspondence: waslon@cear.ufpb.br; Tel.: +55-83-3216-7268
}

Academic Editor: Vittorio M. N. Passaro

Received: 24 August 2016; Accepted: 15 November 2016; Published: 23 November 2016

\begin{abstract}
The performance of signal processing systems based on vector quantization depends on codebook design. In the image compression scenario, the quality of the reconstructed images depends on the codebooks used. In this paper, alternatives are proposed for accelerating families of fuzzy K-means algorithms for codebook design. The acceleration is obtained by reducing the number of iterations of the algorithms and applying efficient nearest neighbor search techniques. Simulation results concerning image vector quantization have shown that the acceleration obtained so far does not decrease the quality of the reconstructed images. Codebook design time savings up to about $40 \%$ are obtained by the accelerated versions with respect to the original versions of the algorithms.
\end{abstract}

Keywords: fuzzy K-means; vector quantization; computational complexity

\section{Introduction}

Signal compression techniques aim at decreasing the number of bits needed to represent the signal (such as speech, image, audio and video), enhancing the efficiency both of transmission and storage. Compression techniques are widely used in applications with storage and bandwidth constraints, such as: storage of medical images, satellite transmissions, voice communication in mobile telephony and videoconference. One of the many techniques used to achieve signal compression is vector quantization (VQ), in which a codebook is used for signal reconstruction.

Vector quantization $[1,2]$ is a lossy compression technique, which uses a mapping $Q$ of a vector $X$, in a $K$-dimensional Euclidean space, into another vector belonging to a finite subset $W$ of $\mathbb{R}^{K}$ :

$$
Q: \mathbb{R}^{K} \rightarrow W
$$

The finite subset $W$ is called a codebook. Each codebook element $w_{j}, 1 \leq j \leq N$, is called a codevector. The number of components in the codevectors is the dimension $(K)$. The size of the codebook is the number of codevectors, denoted by $N$. In several speech coding [3-5] and image coding [6-9] systems, VQ has been used successfully, leading to high compression rates. VQ has also been used in other applications, such as speaker identification $[10,11]$, information security such as steganography and digital watermarking [12-18], and classification of pathological voice signals [19].

Vector quantization is an extension of scalar quantization in a multidimensional space. The performance of VQ depends on the designed codebooks. The prevailing algorithm for codebook design is Linde-Buzo-Gray (LBG) [20], also known as Generalized Lloyd Algorithm (GLA) or K-means. 
Other examples of codebook design algorithms are: fuzzy [7,21,22], competitive learning [23], memetic [24], genetic [25], firefly [26] and honey bee mating optimization [27].

In vector quantization of a digital image, a codebook of size $N$ is used, consisting in $K$-dimensional vectors. The process replaces blocks of pixels from the corresponding image by the most similar blocks of pixels in the codebook. So, the better the codebook, the higher the quantized image quality.

Typical grouping approaches used in VQ split in two categories: crisp and fuzzy clustering. Traditionally, crisp clustering is executed by the K-means algorithm. Due to initialization dependency, $K$-means can be stuck in undesired local minima. On the other hand, fuzzy clustering is usually performed by the fuzzy K-means (FKM) algorithm [28]. FKM attributes each training pattern to every other cluster with different pertinence degrees [29]. Therefore, FKM is able to reduce the random initialization dependency [7,29-31] at a high computational cost.

The K-means (KM) and fuzzy clustering algorithms, e.g., fuzzy K-means (FKM), have been used in a wide range of scenarios and applications, such as: digital soil pattern recognition [32], archaeology [33], indoor localization [34], discrimination of cabernet sauvignon grapevine elements [35], white blood cell segmentation [36], abnormal lung sounds diagnosis [37], intelligent sensor networks in agriculture [38], magnetic resonance image (MRI) segmentation [39,40], speaker recognition [41] and image compression by VQ $[29,42,43]$.

The aforementioned works show that clustering algorithm applications include image coding, biometric authentication, pattern recognition, among others. The performance evaluation of the clustering algorithms depends on the application. In signal compression, an important aspect is the quality of the reconstructed signal. In pattern recognition systems, an important figure of merit is the recognition rate. The processing time of the clustering algorithms is also a relevant aspect. In this paper, techniques are presented for accelerating families of fuzzy K-means algorithms applied to VQ codebook design for image compression. Simulations show that the presented techniques lead to a decrease in processing time for codebook design, while preserving its overall quality.

One of the many techniques used in this work is the Equal-average Nearest Neighbor Search (ENNS) [44,45], which is usually used in the minimum distance coding phase of VQ. However, in this paper, ENNS is used in some of fuzzy K-means families, precisely in the partitioning of the training set. The acceleration of FKM algorithms is also obtained by the use of a lookahead approach in the crisp phase of such algorithms, leading to a decrease in the number of iterations.

The remaining sections are organized as follows: Section 2 covers K-means algorithm and fuzzy K-means families. Section 3 presents modified versions of fuzzy K-means families. In Section 4, nearest neighbor search techniques are introduced with focus in the scenario of accelerating codebook design. The results and final considerations are presented in Sections 5 and 6, respectively.

\section{Codebook Desing Techniques}

Vector quantization performance is highly dependent on codebook quality. The codebook is a set of reference patterns or templates. In digital image coding, the codebook corresponds to a set of reference blocks of pixels. In this paper, K-means algorithm and fuzzy K-means families are the techniques under consideration for codebook design.

The main difference between K-means and fuzzy K-means algorithms is that, in the former, each training vector belongs to one quantization cell. In the latter, each training vector can be associated to more than one quantization cell, with some degree of pertinence to each cell.

$K$-means algorithm partitions the $\mathbb{R}^{K}$ vector space by associating each training vector to a single cluster using nearest neighbor search. Therefore, given an input vector $x_{i}$, it belongs to the cluster (cell or Voronoi region):

$$
V\left(w_{j}\right) \text { if } d\left(x_{i}, w_{j}\right)<d\left(x_{i}, w_{a}\right) \forall a \neq j,
$$

where $d\left(x_{i}, w_{j}\right)$ is a distance measure. Euclidean square distance between $x_{i}$ and $w_{j}$ is widely used in digital image vector quantization. In this case, $w_{j}$ is the nearest neighbor (NN) of $x_{i}$, that is, $w_{j}$ is the quantized version of $x_{i}$. This is equivalent to $w_{j}=Q\left(x_{i}\right)$. The nearest neighbor search can be associated to a pertinence function: 


$$
\mu_{j}\left(x_{i}\right)=\left\{\begin{array}{c}
1, \text { if } w_{j}=\mathrm{NN}\left(x_{i}\right) \\
0, \text { otherwise }
\end{array}\right.
$$

The distortion, obtained by representing the training vectors by their corresponding nearest neighbors, is:

$$
J_{1}=\sum_{j=1}^{N} \sum_{i=1}^{M} \mu_{j}\left(x_{i}\right) d\left(x_{i}, w_{j}\right),
$$

in which $x_{i}$ is the $i$-th training vector, $1 \leq i \leq M$. As $J_{1}$ is a function of $w_{j}$, in order to minimize the distortion, vectors $w_{j}$ are updated according to:

$$
w_{j}=\frac{\sum_{i=1}^{M} \mu_{j}\left(x_{i}\right) x_{i}}{\sum_{i=1}^{M} \mu_{j}\left(x_{i}\right)}, \forall j=1,2, \ldots, N
$$

Equations (2) and (5) are related to the partitioning of the training set and to the codebook update. The algorithm stops at the end of the $n$-th iteration if:

$$
\frac{J_{1}(n-1)-J_{1}(n)}{J_{1}(n)} \leq \varepsilon .
$$

The input parameters of the $K$-means algorithm are: codebook size $(N)$, codevectors dimension $(K)$ and a distortion threshold $\varepsilon$ used as stop criterion.

The fuzzy K-means algorithm aims at minimizing the distortion between training vectors $x_{i}$ and codevectors $w_{j}$ which compose the codebook. Unlike K-means algorithm, fuzzy K-means measures the distortion by [29]:

$$
J_{m}=\sum_{j=1}^{N} \sum_{i=1}^{M} \mu_{j}\left(x_{i}\right)^{m} d\left(x_{i}, w_{j}\right), 1<m<\infty,
$$

subject to the following conditions:

$$
\left\{\begin{array}{c}
\mu_{j}\left(x_{i}\right) \in[0,1] \forall i, j \\
0<\sum_{i=1}^{M} \mu_{j}\left(x_{i}\right)<M \\
\sum_{j=1}^{N} \mu_{j}\left(x_{i}\right)=1, \forall i=1,2, \ldots, M
\end{array}\right.
$$

As stated in [29], $J_{m}$ function minimization results:

$$
\mu_{j}\left(x_{i}\right)=\frac{1}{\sum_{l=1}^{N}\left(\frac{d\left(x_{i}, w_{j}\right)}{d\left(x_{i}, w_{l}\right)}\right)^{\frac{1}{m-1}}} .
$$

Therefore, for a given pertinence degree set of functions, the codevectors evolve at each iteration to minimize $J_{m}$, according to [29]:

$$
w_{j}=\frac{\sum_{i=1}^{M} \mu_{j}\left(x_{i}\right)^{m} x_{i}}{\sum_{i=1}^{M} \mu_{j}\left(x_{i}\right)^{m}}, \forall j=1,2, \ldots, N .
$$

The nebulosity at clusters transitions is controlled by parameter $m$ and increases with this parameter.

The input parameters of the FKM algorithm are: the codebook size $(N)$, the codevector dimension $(K)$, the nebulosity control parameter $m \in(1, \infty)$, and the distortion threshold $\varepsilon$.

This work uses two fuzzy K-means families, as proposed in [29]. The development of those algorithms is based on transition from fuzzy to crisp mode, being the latter mode equivalent to K-means algorithm strategy. The algorithm fuzzy 1 (FKM1) presents three modifications in its construction when compared to FKM. The first is how the pertinence function is calculated: 


$$
\mu_{j}\left(x_{i}\right)=f\left(d\left(x_{i}, w_{j}\right), d_{\max }\left(x_{i}\right)\right)=\left(1-\frac{d\left(x_{i}, w_{j}\right)}{d_{\max }\left(x_{i}\right)}\right)^{u},
$$

in which $d_{\max }\left(x_{i}\right)$ gives the maximum distance between the training vectors and codevectors, and $u$ is a positive integer. The second modification concerns the codebook update, defined by Equation (5). The last modification is found in the transition from fuzzy to crisp mode. For that purpose, a distortion threshold $\varepsilon^{\prime}$ is defined, with $\varepsilon^{\prime}>\varepsilon$. Therefore, FKM1 algorithm has the following parameters as input: $N, K, u$ and two distortion thresholds-precisely, $\varepsilon^{\prime}$ represents the fuzzy to crisp mode transition threshold and $\varepsilon$ represents the stop criterion.

The fuzzy 2 family (FKM2) uses the same codebook update and pertinence function calculations as proposed by fuzzy K-means algorithm, that is, Equations (9) and (10), respectively. The only difference is the inclusion of fuzzy to crisp mode transition.

\section{Accelerating Fuzzy K-Means Family Algorithm}

One of the challenges in the clustering methods is to increase the convergence speed, that is, the decrease in the number of iterations. Some alternatives have been proposed to accelerate K-means algorithm, as the techniques of Lee et al. [46] and Paliwal-Ramasubramanian [47]. Both techniques recalculate the codevectors at the end of each iteration, according to the expression:

$$
w_{j}^{n+1}=w_{j}^{n}+s\left(C\left(V\left(w_{j}^{n}\right)\right)-w_{j}^{n}\right),
$$

where $w_{j}^{n}$ is the codevector at the $n$-th iteration, $s$ is the scale and $C\left(V\left(w_{j}^{n}\right)\right)$ is the centroid of the Voronoi region $V\left(w_{j}^{n}\right)$. Fixed scale $s$ is used in [46]. The modification introduced in [46], proposed in [47], consists in using a scale $s$ which depends on the iteration $n$, that is:

$$
s=1+\frac{v}{v+n},
$$

for some $v>0$.

In this paper, the fuzzy K-Means families accelerated version uses Equations (12) and (13) in codevectors updating. According to simulation results, for FKM1 and FKM2 algorithms, the scale $s$ leads to savings in the number of iterations when applied to the crisp phase of the algorithms.

\section{Nearest Neighbor Search Techniques for Accelerating the Codebook Design}

When FKM1 and FKM2 algorithms change to crisp mode (which is equivalent to the conventional $K$-means algorithm), the complexity of the nearest neighbor search, performed by the $K$-means, can be minimized by efficient search techniques. Usually, K-means algorithm uses Full Search (FS) to compute the nearest neighbor, which is highly time consuming.

A great number of operations can be saved by eliminating poor codevector candidates to the nearest neighbor. This can be accomplished by using search techniques, such as Partial Distortion Search (PDS) [48] and Equal-average Nearest Neighbor Search (ENNS) [44,45]. Both were originally proposed to VQ encoding phase. Instead, in this paper, they are used in FKM1 and FKM2 algorithms. PDS and ENNS apply rejection criteria on codevectors, decreasing, by that means, the time spent in the nearest neighbor search.

PDS algorithm, as proposed in [48], consists of a traditional technique to computational complexity reduction involved in nearest neighbor search. PDS determines, for any $q \leq K$, if the accumulated distance to the first $q$ codevector components is greater than $d_{\min }$ (the minimum distance found in the search so far). If the condition is true, that codevector does not represent the NN. So, it is assumed that the following expression is satisfied:

$$
\sum_{l=1}^{q}\left(x_{i l}-w_{j l}\right)^{2} \geq d_{\text {min }}
$$


where $1 \leq q \leq K, x_{i l}$ is the $l$-th component of training (input) vector $x_{i}$ and $w_{j l}$ is the $l$-th component of codevector $w_{j}$. When this condition is satisfied there is no need to perform the hole calculation for the Euclidean distance between $x_{i}$ and $w_{j}$. With this approach, the number of multiplications, subtractions and additions is reduced, decreasing the search time and, therefore, accelerating the codebook design in comparison to the full search.

In the ENNS algorithm, the mean for each codevector is calculated and sorted previously. Then, a lookup is performed, using some search algorithm, to find the codevector with mean closest to the mean $m_{x}$ of the current input vector $x$. When such codevector is found, searches do not need to be performed for codevectors whose means $m_{i}$ satisfy the criterion:

$$
m_{i} \geq m_{x}+\sqrt{\frac{d_{\text {min }}}{K}} \text { or } m_{i} \leq m_{x}-\sqrt{\frac{d_{\text {min }}}{K}},
$$

where $m_{i}$ is the mean of the $i$-th codevector, $m_{x}$ is the mean of current input vector and $d_{\text {min }}$ is the distance between the input vector and the codevector with the nearest mean.

When the elimination criterion is not satisfied for a given vector, it enters in a waiting list to be looked up later. After all winner candidates to that input vector are collected, a search is performed calculating the square Euclidean distance and the PDS is used.

ENNS decreases the computational time compared to the full search with $N$ (codebook size) memory allocations penalty, compared to PDS. That fact is proved in [49]. Because ENNS was originally used in coding phase, it performs one means sorting, since the codebook vectors were previously designed. However, as for the codebook design, the K-means algorithm (on the crisp mode of FKM families), at each iteration, updates its codevectors, hereby a new average sorting is needed for each iteration. Acceleration alternatives in the scenario of FKM2 are presented as follows (see Algorithms 1-3). The notation MFKM2 stands for modified fuzzy K-means family 2, that is, an acceleration (savings in the number of iterations) obtaining by using the scale factor $s$ in codebook update.
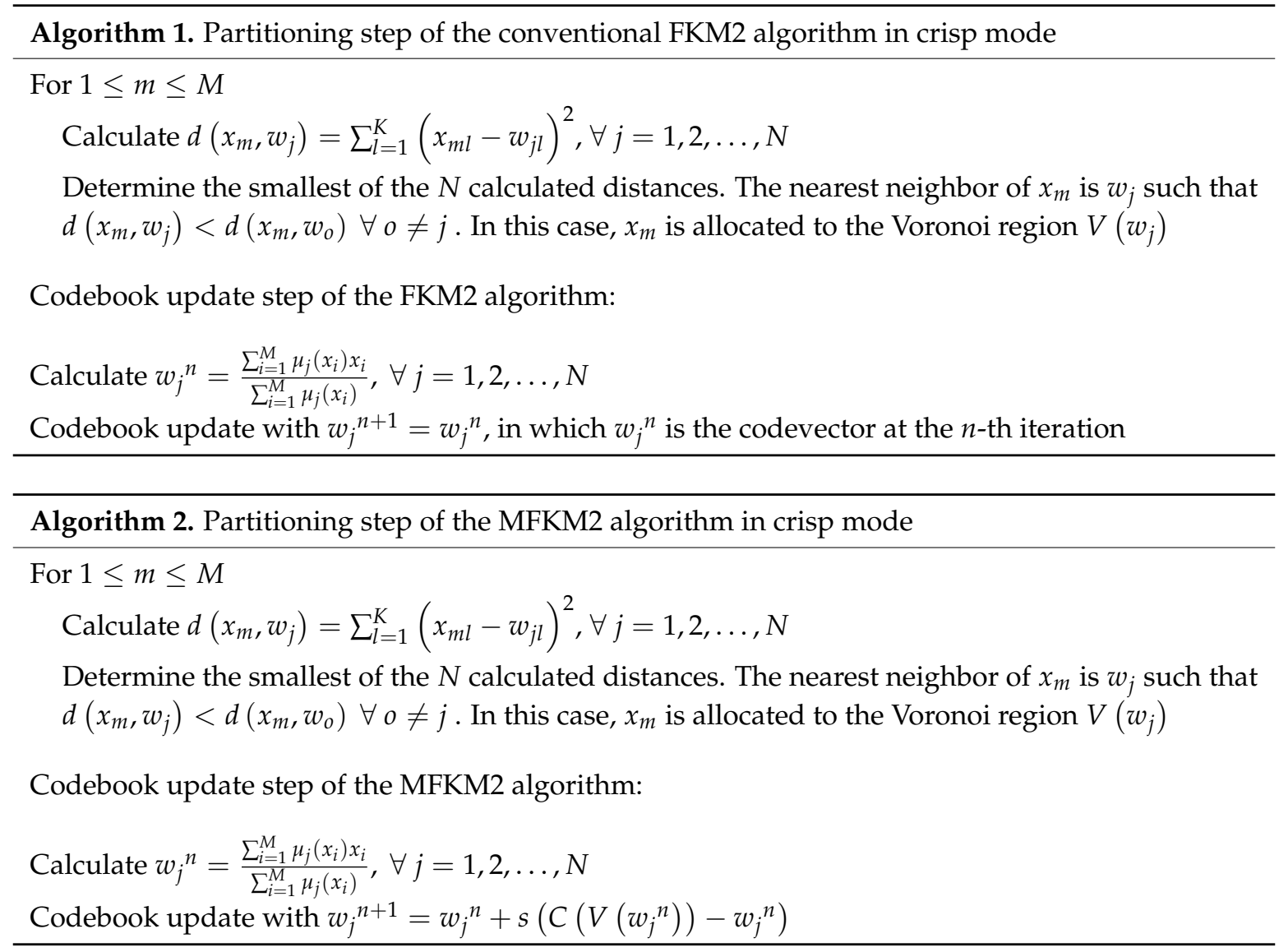
It is worth mentioning that other approaches have been proposed in the literature for the purpose of fast codebook search. As an example, the method introduced by Chang and $\mathrm{Wu}$ [50] is an interesting partial-search technique based on a graph structure which leads to computational cost savings.

\footnotetext{
Algorithm 3. Partitioning step of the MFKM2 algorithm in crisp mode with the use of ENNS
}

(Calculate off-line the mean of each input vector)

Calculate the mean of each codevector and order the $N$ means in ascending order

For $1 \leq m \leq M$

Determine the codevector with the minimum absolute difference between its mean and the input vector mean. Obtain $d_{\min }$ as the squared Euclidean distance between this codevector and the input vector

Eliminate from the search process the codevectors that satisfy:

$$
m_{i} \geq m_{x}+\sqrt{\frac{d_{\min }}{K}} \text { or } m_{i} \leq m_{x}-\sqrt{\frac{d_{\min }}{K}}
$$

For the remaining codevectors, i.e., those who were not eliminated from the search, apply the PDS algorithm for calculating the distance and update $d_{\min }$ (the minimum distance found in the search so far)

At the end of the process, the codevector $w_{j}$ corresponding to $d_{\min }$ is the nearest neighbor of $x_{m}$. In this case, $x_{m}$ is allocated to the Voronoi region $V\left(w_{j}\right)$

Codebook update step of the MFKM2 algorithm:

Calculate $w_{j}{ }^{n}=\frac{\sum_{i=1}^{M} \mu_{j}\left(x_{i}\right) x_{i}}{\sum_{i=1}^{M} \mu_{j}\left(x_{i}\right)}, \forall j=1,2, \ldots, N$

Codebook update with $w_{j}{ }^{n+1}=w_{j}{ }^{n}+s\left(C\left(V\left(w_{j}{ }^{n}\right)\right)-w_{j}{ }^{n}\right)$

\section{Results}

Simulations have been performed in a core I5-2450m $(2.50 \mathrm{GHz})$ Intel computer using nine $256 \times 256$ pixel images: Lena, Barbara, Elaine, Boat, Clock, Goldhill, Peppers, Mandrill and Tiffany. Each image has 256 gray scale levels, as shown in Figure 1. The parameters used for the simulations were: $K=16\left(4 \times 4\right.$ pixel blocks), $N=32,64,128$ and $256, u=2$ and two distortion thresholds, $\varepsilon^{\prime}=0.1$ and $\varepsilon=0.001$. For each parameter combination of dimension $K$ and codebook size $N$ (for example $N=32$ and $K=16$ ), 20 random initializations were used for each algorithm.

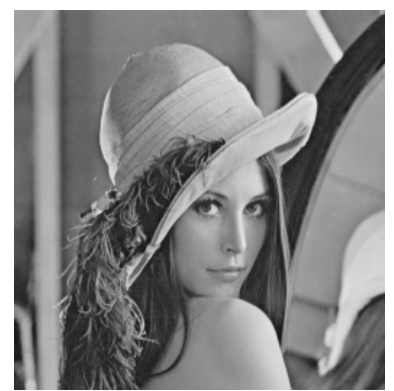

(a)

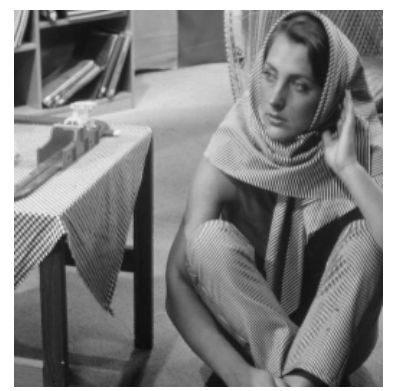

(b)

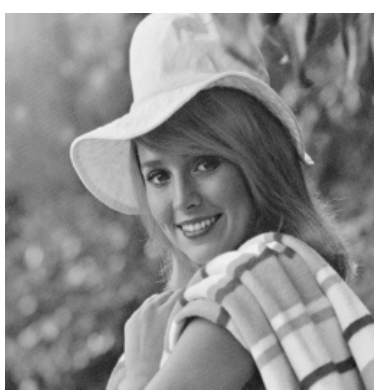

(c)

Figure 1. Cont. 


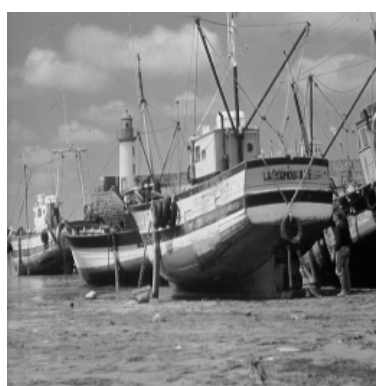

(d)

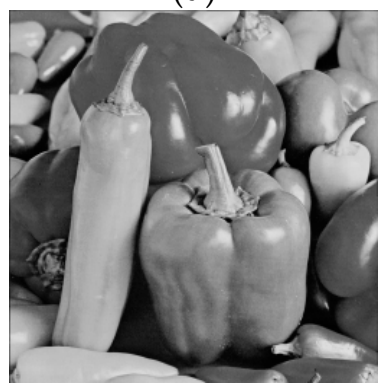

(g)

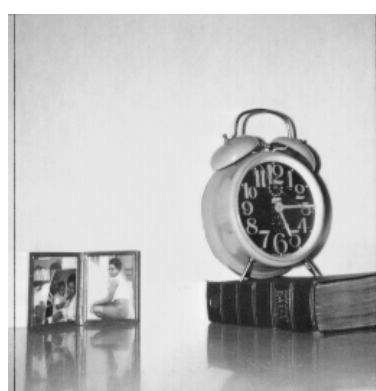

(e)

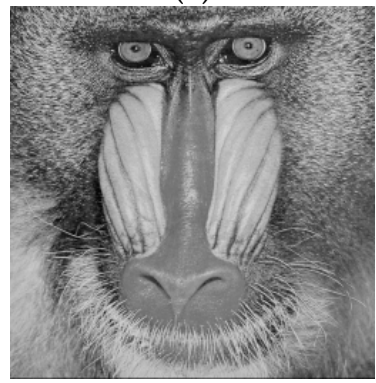

(h)

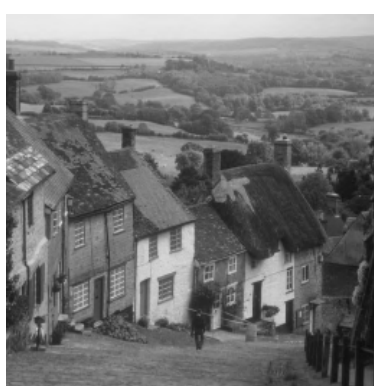

(f)

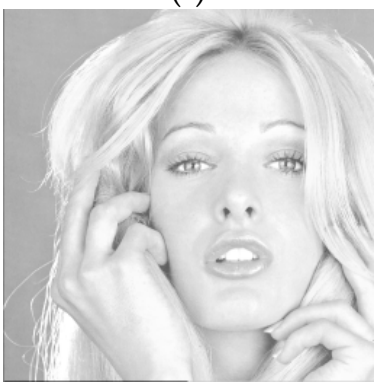

(i)

Figure 1. Images $256 \times 256$ pixels, 8.0 bpp. (a) Lena; (b) Barbara; (c) Elaine; (d) Boat; (e) Clock; (f) Goldhill; (g) Peppers; (h) Mandrill; (i) Tiffany.

Results are presented in terms of average number of iterations and average execution time (in seconds) of the codebook design algorithms, as well as average peak signal noise ratio (PSNR) and structural similarity (SSIM) index [51] of reconstructed images. The notation adopted for the methods are presented in Table 1. Results are organized in Tables 2-15.

Table 1. Notation.

\begin{tabular}{|c|c|}
\hline KM & K-means \\
\hline FKM & Fuzzy K-means \\
\hline MFKM & Modified Fuzzy K-means (accelerated version with scale s) \\
\hline FKM1 & Fuzzy K-means Family 1 \\
\hline MFKM1 & Modified Fuzzy K-means Family 1 (accelerated version with scale $s$ ) \\
\hline FKM1-PDS & Fuzzy K-means Family 1 with Partial Distortion Search in the crisp phase \\
\hline MFKM1-PDS & $\begin{array}{l}\text { Modified Fuzzy K-means Family } 1 \text { (accelerated version with scale } s \text { ) with Partial Distortion } \\
\text { Search in the crisp phase }\end{array}$ \\
\hline FKM1-ENNS & Fuzzy K-means Family 1 with Equal-Average Nearest Neighbor Search in the crisp phase \\
\hline MFKM1-ENNS & $\begin{array}{l}\text { Modified Fuzzy K-means Family } 1 \text { (accelerated version with scale } s \text { ) with Equal-Average } \\
\text { Nearest Neighbor Search in the crisp phase }\end{array}$ \\
\hline FKM2 & Fuzzy K-means Family 2 \\
\hline MFKM2 & Modified Fuzzy K-means Family 2 (accelerated version with scale $s$ ) \\
\hline FKM2-PDS & Fuzzy K-means Family 2 with Partial Distortion Search in the crisp phase \\
\hline MFKM2-PDS & $\begin{array}{l}\text { Modified Fuzzy K-means Family } 2 \text { (accelerated version with scale } s \text { ) with Partial Distortion } \\
\text { Search in the crisp phase }\end{array}$ \\
\hline FKM2-ENNS & Fuzzy K-means Family 2 with Equal-Average Nearest Neighbor Search in the crisp phase \\
\hline MFKM2-ENNS & $\begin{array}{l}\text { Modified Fuzzy K-means Family } 2 \text { (accelerated version with scale } s \text { ) with Equal-Average } \\
\text { Nearest Neighbor Search in the crisp phase }\end{array}$ \\
\hline
\end{tabular}

Regarding Table 2, all algorithms under consideration led to close values of PSNR. It can be noted that the use of the scale factors led to a decrease in the average number of iterations. In other words, 
it is observed, for instance, that the average number of iterations of MFKM is smaller than that of FKM. The decrease in the number of iterations is also observed when one compares MFKM1 with FKM1, as well as when one compares MFKM2 with FKM2. The use of PDS for nearest neighbor search contributes to reduce the time spent for codebook design. For instance, considering Elaine image, for FKM1 and FKM1-PDS, the use of PDS in the partitioning step of the second phase (crisp phase) of FKM1 led to a codebook design average time $0.34 \mathrm{~s}$, which is lower than $0.38 \mathrm{~s}$ spent for codebook design using the full search (FS) or brute force in that phase. If the ENNS is used in substitution to FS, the time spent is $0.27 \mathrm{~s}$. The highest time savings, concerning FKM1, is obtained by using the scale factor $s$ to decrease the number of iterations combined with the use of ENNS for efficient nearest neighbor search. Indeed, regarding Elaine image, that combination led to an average time spent for codebook design equals $0.25 \mathrm{~s}$.

Table 2. PSNR (in dB), number of iterations and codebook design time (in seconds) for images Lena, Barbara and Elaine, using $N=32$.

\begin{tabular}{cccccccccc}
\hline \multirow{2}{*}{ Algorithm } & \multicolumn{3}{c}{ Lena } & \multicolumn{3}{c}{ Barbara } & \multicolumn{3}{c}{ Elaine } \\
\cline { 2 - 10 } & PSNR & Iter & Time & PSNR & Iter & Time & PSNR & Iter & Time \\
\hline KM & 26.61 & 17.20 & 0.16 & 24.76 & 15.20 & 0.12 & 27.75 & 18.15 & 0.16 \\
FKM & 26.57 & 19.65 & 1.53 & 24.71 & 16.00 & 1.11 & 27.70 & 19.75 & 1.65 \\
MFKM & 26.61 & 14.75 & 1.12 & 24.72 & 12.30 & 0.86 & 27.72 & 15.80 & 1.31 \\
FKM1 & 26.60 & 22.35 & 0.35 & 24.77 & 19.00 & 0.38 & 27.77 & 24.35 & 0.38 \\
MFKM1 & 26.62 & 18.25 & 0.28 & 24.79 & 16.05 & 0.31 & 27.77 & 18.55 & 0.30 \\
FKM1-PDS & 26.60 & 22.35 & 0.33 & 24.77 & 19.00 & 0.34 & 27.77 & 24.35 & 0.34 \\
MFKM1-PDS & 26.62 & 18.25 & 0.26 & 24.79 & 16.05 & 0.28 & 27.77 & 18.55 & 0.29 \\
FKM1-ENNS & 26.60 & 22.35 & 0.26 & 24.77 & 19.00 & 0.28 & 27.77 & 24.35 & 0.27 \\
MFKM1-ENNS & 26.62 & 18.25 & 0.22 & 24.79 & 16.05 & 0.25 & 27.77 & 18.55 & 0.25 \\
FKM2 & 26.60 & 15.35 & 0.39 & 24.77 & 14.25 & 0.30 & 27.77 & 18.50 & 0.40 \\
MFKM2 & 26.63 & 12.70 & 0.35 & 24.78 & 11.75 & 0.24 & 27.80 & 14.40 & 0.33 \\
FKM2-PDS & 26.60 & 15.35 & 0.35 & 24.77 & 14.25 & 0.27 & 27.77 & 18.45 & 0.37 \\
MFKM2-PDS & 26.63 & 12.70 & 0.33 & 24.78 & 11.75 & 0.22 & 27.80 & 14.40 & 0.30 \\
FKM2-ENNS & 26.60 & 15.35 & 0.33 & 24.77 & 14.25 & 0.24 & 27.77 & 18.50 & 0.29 \\
MFKM2-ENNS & 26.63 & 12.70 & 0.30 & 24.78 & 11.75 & 0.20 & 27.80 & 14.40 & 0.26 \\
\hline
\end{tabular}

With respect to Table 3, it is observed that the highest time spent for codebook design was for FKM algorithm. It is important to mention that this behavior is observed for all images and codebook sizes considered in the present work. As an example, for the Boat image and codebook size $N=32$, the codebook design average time spent by FKM is $1.64 \mathrm{~s}$, which is 8.2 times higher than the average time spent by KM and about 3.8 times higher than the average time spent by FKM2. Table 3 results also confirm the benefits of using the modified versions of the codebook design algorithms (M versions, with the use of the scale factor $s$ ) and nearest search algorithms for codebook design time savings when compared to the standard versions of the codebook design algorithms. For each image under consideration, it is observed that all algorithms lead to close PSNR values.

From the results presented in Tables 4 and 5, it is observed that the codebook design average time spent by FKM2 is higher than that one of FKM1. It is important to mention that the same behavior is observed for all the images under consideration, for codebook sizes 128 and 256. Regarding the number of iterations, it is observed in Tables 4 and 5 that the modified versions with the use of the scale factor $s$ (algorithms MFKM, MFKM1 and MFKM2) have and average execution time lower than that of the corresponding standard versions (FKM, FKM1 and FKM2 respectively) - due to the savings in the number of iterations. Tables 4 and 5 point out that the lowest codebook design average time is obtained with the combination of the scale factor $s$ and ENNS. Indeed, considering for instance fuzzy K-means family 2 and Clock image, in Table 5 the average time of MFKM2-ENNS is $0.92 \mathrm{~s}$, which is lower than the average time presented by all the other versions (FKM2, MFKM2, FKM2-PDS, MFKM2-PDS and FKM2-ENNS). 
Table 3. PSNR (in dB), number of iterations and codebook design time (in seconds) for images Boat, Clock and Goldhill, using $N=32$.

\begin{tabular}{cccccccccc}
\hline \multirow{2}{*}{ Algorithm } & \multicolumn{3}{c}{ Boat } & \multicolumn{3}{c}{ Clock } & \multicolumn{3}{c}{ Goldhill } \\
\cline { 2 - 9 } & PSNR & Iter & Time & PSNR & Iter & Time & PSNR & Iter & Time \\
\hline KM & 24.92 & 18.95 & 0.20 & 26.16 & 26.10 & 0.32 & 26.66 & 17.00 & 0.34 \\
FKM & 24.84 & 21.20 & 1.64 & 26.23 & 41.00 & 1.78 & 26.67 & 18.60 & 3.05 \\
MFKM & 24.87 & 16.20 & 0.98 & 26.28 & 35.55 & 1.08 & 26.70 & 16.30 & 2.57 \\
FKM1 & 24.91 & 25.75 & 0.42 & 26.19 & 33.40 & 0.55 & 26.67 & 22.25 & 0.46 \\
MFKM1 & 24.93 & 19.80 & 0.33 & 26.25 & 25.80 & 0.45 & 26.68 & 18.85 & 0.36 \\
FKM1-PDS & 24.91 & 25.75 & 0.40 & 26.19 & 33.40 & 0.50 & 26.67 & 22.25 & 0.43 \\
MFKM1-PDS & 24.93 & 19.80 & 0.31 & 26.25 & 25.80 & 0.41 & 26.68 & 18.85 & 0.32 \\
FKM1-ENNS & 24.91 & 25.75 & 0.32 & 26.19 & 33.40 & 0.39 & 26.67 & 22.25 & 0.35 \\
MFKM1-ENNS & 24.93 & 19.80 & 0.27 & 26.25 & 25.80 & 0.34 & 26.68 & 18.85 & 0.30 \\
FKM2 & 24.91 & 18.90 & 0.43 & 26.26 & 23.30 & 0.46 & 26.67 & 16.20 & 0.63 \\
MFKM2 & 24.93 & 15.20 & 0.37 & 26.32 & 20.50 & 0.40 & 26.70 & 13.55 & 0.55 \\
FKM2-PDS & 24.91 & 18.90 & 0.41 & 26.26 & 23.30 & 0.43 & 26.67 & 16.20 & 0.58 \\
MFKM2-PDS & 24.93 & 15.20 & 0.35 & 26.32 & 20.50 & 0.39 & 26.70 & 13.55 & 0.51 \\
FKM2-ENNS & 24.91 & 18.90 & 0.36 & 26.26 & 23.30 & 0.40 & 26.67 & 16.20 & 0.47 \\
MFKM2-ENNS & 24.93 & 15.20 & 0.32 & 26.32 & 20.50 & 0.34 & 26.70 & 13.55 & 0.46 \\
\hline
\end{tabular}

Table 4. PSNR (in dB), number of iterations and codebook design time (in seconds) for images Lena, Barbara and Elaine, using $N=64$.

\begin{tabular}{cccccccccc}
\hline \multirow{2}{*}{ Algorithm } & \multicolumn{3}{c}{ Lena } & \multicolumn{3}{c}{ Barbara } & \multicolumn{3}{c}{ Elaine } \\
\cline { 2 - 9 } & PSNR & Iter & Time & PSNR & Iter & Time & PSNR & Iter & Time \\
\hline KM & 27.74 & 17.80 & 0.32 & 25.68 & 16.25 & 0.25 & 29.06 & 18.00 & 0.24 \\
FKM & 27.69 & 22.85 & 5.81 & 25.64 & 21.15 & 4.03 & 29.09 & 24.05 & 4.91 \\
MFKM & 27.73 & 17.90 & 4.51 & 25.64 & 15.40 & 3.31 & 29.13 & 18.60 & 3.98 \\
FKM1 & 27.67 & 23.15 & 0.61 & 25.74 & 20.65 & 0.55 & 29.01 & 23.55 & 0.62 \\
MFKM1 & 27.75 & 19.10 & 0.48 & 25.77 & 17.95 & 0.48 & 29.07 & 19.90 & 0.53 \\
FKM1-PDS & 27.67 & 23.15 & 0.53 & 25.74 & 20.65 & 0.51 & 29.01 & 23.55 & 0.54 \\
MFKM1-PDS & 27.75 & 19.10 & 0.46 & 25.77 & 17.95 & 0.44 & 29.07 & 19.90 & 0.47 \\
FKM1-ENNS & 27.67 & 23.15 & 0.39 & 25.74 & 20.65 & 0.43 & 29.01 & 23.55 & 0.46 \\
MFKM1-ENNS & 27.75 & 19.10 & 0.35 & 25.77 & 17.95 & 0.36 & 29.07 & 19.90 & 0.40 \\
FKM2 & 27.80 & 14.50 & 0.81 & 25.73 & 15.00 & 0.71 & 29.05 & 16.45 & 0.81 \\
MFKM2 & 27.85 & 12.85 & 0.71 & 25.75 & 12.85 & 0.61 & 29.10 & 13.70 & 0.75 \\
FKM2-PDS & 27.80 & 14.50 & 0.70 & 25.73 & 15.00 & 0.62 & 29.05 & 16.45 & 0.74 \\
MFKM2-PDS & 27.85 & 12.85 & 0.67 & 25.75 & 12.85 & 0.55 & 29.10 & 13.70 & 0.68 \\
FKM2-ENNS & 27.80 & 14.50 & 0.62 & 25.73 & 14.95 & 0.57 & 29.05 & 16.40 & 0.62 \\
MFKM2-ENNS & 27.85 & 12.85 & 0.60 & 25.75 & 12.85 & 0.52 & 29.10 & 13.70 & 0.60 \\
\hline
\end{tabular}

Table 5. PSNR (in dB), number of iterations and codebook design time (in seconds) for images Boat, Clock and Goldhill, using $N=64$.

\begin{tabular}{cccccccccc}
\hline \multirow{2}{*}{ Algorithm } & \multicolumn{3}{c}{ Boat } & \multicolumn{3}{c}{ Clock } & \multicolumn{3}{c}{ Goldhill } \\
\cline { 2 - 10 } & PSNR & Iter & Time & PSNR & Iter & Time & PSNR & Iter & Time \\
\hline KM & 25.90 & 18.45 & 0.46 & 27.17 & 22.05 & 0.62 & 27.69 & 16.15 & 0.36 \\
FKM & 25.84 & 23.30 & 6.31 & 27.41 & 42.70 & 8.11 & 27.68 & 19.55 & 5.81 \\
MFKM & 25.85 & 16.05 & 4.61 & 27.46 & 33.85 & 5.53 & 27.70 & 15.20 & 4.51 \\
FKM1 & 25.85 & 24.35 & 0.73 & 27.08 & 25.10 & 1.05 & 27.69 & 21.80 & 0.64 \\
MFKM1 & 25.91 & 18.90 & 0.62 & 27.16 & 20.10 & 0.80 & 27.71 & 18.05 & 0.53 \\
FKM1-PDS & 25.85 & 24.35 & 0.68 & 27.08 & 25.65 & 0.88 & 27.69 & 21.85 & 0.60 \\
MFKM1-PDS & 25.91 & 18.90 & 0.56 & 27.16 & 20.10 & 0.70 & 27.71 & 18.05 & 0.49 \\
FKM1-ENNS & 25.85 & 24.35 & 0.55 & 27.08 & 25.10 & 0.68 & 27.69 & 21.80 & 0.48 \\
MFKM1-ENNS & 25.91 & 18.90 & 0.44 & 27.16 & 20.10 & 0.52 & 27.71 & 18.05 & 0.43 \\
FKM2 & 25.92 & 17.50 & 0.94 & 27.32 & 18.90 & 1.09 & 27.70 & 15.60 & 1.04 \\
MFKM2 & 25.96 & 13.80 & 0.85 & 27.40 & 16.10 & 1.01 & 27.73 & 13.10 & 0.89 \\
FKM2-PDS & 25.92 & 17.45 & 0.87 & 27.32 & 18.90 & 1.03 & 27.70 & 15.55 & 0.96 \\
MFKM2-PDS & 25.96 & 13.80 & 0.83 & 27.40 & 16.10 & 0.98 & 27.73 & 13.10 & 0.93 \\
FKM2-ENNS & 25.92 & 17.50 & 0.84 & 27.32 & 18.90 & 0.94 & 27.70 & 15.60 & 0.83 \\
MFKM2-ENNS & 25.96 & 13.80 & 0.70 & 27.40 & 16.10 & 0.92 & 27.73 & 13.10 & 0.75 \\
\hline
\end{tabular}


It is observed in Tables $2-5$ that the best PSNR results, for five out of six images under consideration, for $N=32$ and $N=64$, are obtained by using algorithms MFKM2, MFKM2-PDS and MFKM2-ENNS.

From Tables 6 and 7, for all images under consideration and for all codebook sizes, the modified versions (those using the scale factor $s$ ) of the algorithms led to average number of iterations smaller than that of the original versions. For instance, for Lena image, the average number of iterations of MFKM is 21.25 and the corresponding number of FKM is 27.60; for Goldhill image, MFKM1 average number of iterations is 16.30 and FKM1 average number of iterations is 20.60; for Boat image, the average number of iterations of MFKM2 is 14.15, and the corresponding number of FKM2 is 16.25. The use of ENNS has proved to be an effective alternative for codebook design time savings. Consider, for instance, Elaine image, for which the codebook design average time of FKM1-ENNS is $0.77 \mathrm{~s}$, while the corresponding time for FKM1 is $1.13 \mathrm{~s}$. For all images under consideration, for each family of fuzzy K-means algorithm, the highest codebook design time savings is obtained by combining the use of scale factor $s$ (M version of the codebook design algorithm) with ENNS. As an example, for all images under consideration, the codebook design average time spent by MFKM2-ENNS is lower than the corresponding one of FKM2, MFKM2, FKM2-PDS, MFKM2-PDS and FKM2-ENNS.

Table 6. PSNR (in dB), number of iterations and codebook design time (in seconds) for images Lena, Barbara and Elaine, using $N=128$.

\begin{tabular}{cccccccccc}
\hline \multirow{2}{*}{ Algorithm } & \multicolumn{3}{c}{ Lena } & \multicolumn{3}{c}{ Barbara } & \multicolumn{3}{c}{ Elaine } \\
\cline { 2 - 9 } & PSNR & Iter & Time & PSNR & Iter & Time & PSNR & Iter & Time \\
\hline KM & 28.83 & 18.10 & 0.51 & 26.68 & 14.95 & 0.45 & 30.27 & 16.30 & 0.51 \\
FKM & 28.91 & 27.60 & 22.31 & 26.61 & 20.35 & 13.45 & 30.40 & 26.15 & 18.47 \\
MFKM & 28.95 & 21.25 & 16.32 & 26.64 & 16.70 & 11.57 & 30.44 & 19.50 & 13.11 \\
FKM1 & 28.73 & 22.20 & 1.10 & 26.74 & 20.80 & 1.06 & 30.17 & 23.10 & 1.13 \\
MFKM1 & 28.92 & 17.55 & 0.91 & 26.81 & 16.05 & 0.85 & 30.30 & 18.55 & 0.91 \\
FKM1-PDS & 28.73 & 22.25 & 0.96 & 26.74 & 20.90 & 0.96 & 30.17 & 23.10 & 1.05 \\
MFKM1-PDS & 28.92 & 17.55 & 0.81 & 26.81 & 15.95 & 0.75 & 30.30 & 18.55 & 0.82 \\
FKM1-ENNS & 28.73 & 22.20 & 0.79 & 26.74 & 20.80 & 0.77 & 30.17 & 23.10 & 0.77 \\
MFKM1-ENNS & 28.92 & 17.55 & 0.68 & 26.81 & 16.05 & 0.63 & 30.30 & 18.55 & 0.68 \\
FKM2 & 28.97 & 14.45 & 1.97 & 26.74 & 14.30 & 1.60 & 30.34 & 14.35 & 1.83 \\
MFKM2 & 29.07 & 12.55 & 1.76 & 26.79 & 12.85 & 1.54 & 30.45 & 12.70 & 1.73 \\
FKM2-PDS & 28.97 & 14.45 & 1.76 & 26.74 & 14.30 & 1.55 & 30.34 & 14.35 & 1.72 \\
MFKM2-PDS & 29.07 & 12.55 & 1.65 & 26.79 & 12.85 & 1.48 & 30.45 & 12.70 & 1.66 \\
FKM2-ENNS & 28.97 & 14.45 & 1.60 & 26.74 & 14.30 & 1.47 & 30.34 & 14.30 & 1.59 \\
MFKM2-ENNS & 29.07 & 12.55 & 1.56 & 26.79 & 12.85 & 1.39 & 30.45 & 12.70 & 1.57 \\
\hline
\end{tabular}

Table 7. PSNR (in dB), number of iterations and codebook design time (in seconds) for images Boat, Clock and Goldhill, using $N=128$.

\begin{tabular}{cccccccccc}
\hline \multirow{2}{*}{ Algorithm } & \multicolumn{3}{c}{ Boat } & & & Clock & & \multicolumn{3}{c}{ Goldhill } \\
\cline { 2 - 9 } & PSNR & Iter & Time & PSNR & Iter & Time & PSNR & Iter & Time \\
\hline KM & 26.90 & 17.80 & 0.53 & 28.28 & 16.60 & 0.65 & 28.67 & 15.05 & 0.41 \\
FKM & 26.91 & 26.85 & 25.38 & 28.48 & 31.40 & 39.61 & 28.66 & 20.30 & 13.51 \\
MFKM & 26.94 & 20.70 & 22.56 & 28.55 & 26.05 & 36.47 & 28.69 & 15.35 & 11.02 \\
FKM1 & 26.59 & 24.15 & 1.22 & 28.04 & 20.50 & 1.36 & 28.69 & 20.60 & 1.15 \\
MFKM1 & 26.70 & 17.20 & 0.98 & 28.24 & 17.50 & 1.13 & 28.77 & 16.30 & 0.99 \\
FKM1-PDS & 26.59 & 24.15 & 1.17 & 28.04 & 20.35 & 1.26 & 28.69 & 20.75 & 1.02 \\
MFKM1-PDS & 26.70 & 17.20 & 0.93 & 28.24 & 17.50 & 1.03 & 28.77 & 16.30 & 0.95 \\
FKM1-ENNS & 26.59 & 24.15 & 0.90 & 28.04 & 20.50 & 0.85 & 28.69 & 20.60 & 0.90 \\
MFKM1-ENNS & 26.70 & 17.20 & 0.72 & 28.24 & 17.50 & 0.73 & 28.77 & 16.30 & 0.83 \\
FKM2 & 26.97 & 16.25 & 2.04 & 28.28 & 14.40 & 2.56 & 28.69 & 14.30 & 1.80 \\
MFKM2 & 27.07 & 14.15 & 1.85 & 28.40 & 13.15 & 2.48 & 28.75 & 12.95 & 1.75 \\
FKM2-PDS & 26.97 & 16.25 & 1.97 & 28.28 & 14.40 & 2.47 & 28.69 & 14.30 & 1.76 \\
MFKM2-PDS & 27.07 & 14.15 & 1.76 & 28.40 & 13.15 & 2.45 & 28.75 & 12.95 & 1.70 \\
FKM2-ENNS & 26.97 & 16.25 & 1.77 & 28.28 & 14.40 & 2.32 & 28.69 & 14.35 & 1.64 \\
MFKM2-ENNS & 27.07 & 14.15 & 1.68 & 28.40 & 13.15 & 2.30 & 28.75 & 12.95 & 1.52 \\
\hline
\end{tabular}


As can be observed in Tables 8 and 9, in comparison with FKM1 family, the modified version MFKM1 has a smaller average number of iterations, which lead to a lower codebook design average time. Additional time savings is obtained by the use of efficient nearest neighbor search methods, that is, PDS or ENNS. It is important to observe that the modified versions generally lead to higher PSNR values when compared to the original versions. As an example, for Lena image, MFKM1 led to $30.13 \mathrm{~dB}$ average PSNR, while the original version led to a corresponding 29.74 dB PSNR; for the same image, the substitution of FKM2 by MFKM2 led to an increase of $0.20 \mathrm{~dB}$ in terms of average PSNR.

Table 8. PSNR (in dB), number of iterations and codebook design time (in seconds) for images Lena, Barbara and Elaine, using $N=256$.

\begin{tabular}{cccccccccc}
\hline \multirow{2}{*}{ Algorithm } & \multicolumn{3}{c}{ Lena } & \multicolumn{3}{c}{ Barbara } & \multicolumn{3}{c}{ Elaine } \\
\cline { 2 - 9 } & PSNR & Iter & Time & PSNR & Iter & Time & PSNR & Iter & Time \\
\hline KM & 29.89 & 14.70 & 0.62 & 27.76 & 13.60 & 0.58 & 31.46 & 14.40 & 0.66 \\
FKM & 30.21 & 38.20 & 90.19 & 27.74 & 25.25 & 66.32 & 31.80 & 31.65 & 79.91 \\
MFKM & 30.24 & 27.10 & 73.28 & 27.76 & 20.00 & 57.34 & 31.87 & 24.30 & 75.85 \\
FKM1 & 29.74 & 21.80 & 1.99 & 27.78 & 18.40 & 1.89 & 31.16 & 20.65 & 1.97 \\
MFKM1 & 30.13 & 16.20 & 1.78 & 27.97 & 15.15 & 1.71 & 31.53 & 17.10 & 1.75 \\
FKM1-PDS & 29.74 & 21.80 & 1.78 & 27.78 & 18.40 & 1.74 & 31.16 & 20.65 & 1.73 \\
MFKM1-PDS & 30.13 & 16.20 & 1.56 & 27.97 & 15.15 & 1.59 & 31.53 & 17.10 & 1.52 \\
FKM1-ENNS & 29.74 & 21.75 & 1.40 & 27.78 & 18.50 & 1.41 & 31.16 & 20.75 & 1.46 \\
MFKM1-ENNS & 30.13 & 16.20 & 1.36 & 27.97 & 15.15 & 1.40 & 31.53 & 17.10 & 1.38 \\
FKM2 & 30.23 & 14.10 & 5.04 & 27.88 & 13.35 & 5.10 & 31.65 & 13.10 & 5.32 \\
MFKM2 & 30.43 & 12.75 & 5.17 & 28.00 & 12.05 & 5.04 & 31.77 & 12.25 & 5.79 \\
FKM2-PDS & 30.23 & 14.10 & 4.92 & 27.88 & 13.35 & 4.81 & 31.65 & 13.10 & 5.16 \\
MFKM2-PDS & 30.43 & 12.75 & 5.22 & 28.00 & 12.05 & 4.65 & 31.77 & 12.25 & 5.42 \\
FKM2-ENNS & 30.23 & 14.10 & 4.63 & 27.88 & 13.30 & 4.65 & 31.65 & 13.10 & 4.92 \\
MFKM2-ENNS & 30.43 & 12.75 & 4.94 & 28.00 & 12.05 & 4.58 & 31.77 & 12.25 & 5.37 \\
\hline
\end{tabular}

Table 9. PSNR (in dB), number of iterations and codebook design time (in seconds) for images Boat, Clock and Goldhill, using $N=256$.

\begin{tabular}{cccccccccc}
\hline \multirow{2}{*}{ Algorithm } & \multicolumn{3}{c}{ Boat } & \multicolumn{3}{c}{ Clock } & \multicolumn{3}{c}{ Goldhill } \\
\cline { 2 - 9 } & PSNR & Iter & Time & PSNR & Iter & Time & PSNR & Iter & Time \\
\hline KM & 27.91 & 13.30 & 0.63 & 29.47 & 13.70 & 0.65 & 29.73 & 13.30 & 0.61 \\
FKM & 28.04 & 32.05 & 84.26 & 29.82 & 35.65 & 81.15 & 29.83 & 24.70 & 63.14 \\
MFKM & 28.08 & 23.65 & 70.18 & 29.85 & 26.00 & 75.34 & 29.86 & 18.50 & 54.12 \\
FKM1 & 27.57 & 24.05 & 2.44 & 29.09 & 19.75 & 1.81 & 29.68 & 19.30 & 2.12 \\
MFKM1 & 27.87 & 17.55 & 1.93 & 29.41 & 16.10 & 1.59 & 29.90 & 15.80 & 1.88 \\
FKM1-PDS & 27.57 & 24.05 & 2.25 & 29.09 & 19.75 & 1.64 & 29.68 & 19.30 & 1.96 \\
MFKM1-PDS & 27.87 & 17.55 & 1.79 & 29.41 & 16.10 & 1.42 & 29.90 & 15.80 & 1.76 \\
FKM1-ENNS & 27.57 & 23.95 & 1.77 & 29.09 & 19.70 & 1.29 & 29.68 & 19.35 & 1.51 \\
MFKM1-ENNS & 27.87 & 17.55 & 1.43 & 29.41 & 16.10 & 1.12 & 29.90 & 15.80 & 1.42 \\
FKM2 & 28.05 & 13.40 & 5.29 & 29.56 & 12.75 & 5.10 & 29.80 & 12.10 & 5.12 \\
MFKM2 & 28.22 & 11.85 & 5.53 & 29.75 & 12.40 & 5.35 & 29.92 & 11.80 & 5.62 \\
FKM2-PDS & 28.05 & 13.40 & 5.09 & 29.56 & 12.75 & 4.82 & 29.80 & 12.10 & 5.05 \\
MFKM2-PDS & 28.22 & 11.85 & 5.15 & 29.75 & 12.40 & 5.12 & 29.92 & 11.80 & 5.34 \\
FKM2-ENNS & 28.05 & 13.40 & 4.76 & 29.56 & 12.75 & 4.52 & 29.80 & 12.10 & 4.83 \\
MFKM2-ENNS & 28.22 & 11.85 & 5.06 & 29.75 & 12.40 & 4.50 & 29.92 & 11.80 & 5.21 \\
\hline
\end{tabular}

According to Tables 8 and 9, for codebook size $N=256$, for four out of six images under consideration, the best PSNR results are obtained by using algorithms MFKM2, MFKM2-PDS and MFKM2-ENNS. Particularly, for Lena image, the substitution of KM by MFKM2-ENNS lead to a PSNR gain of $0.54 \mathrm{~dB}$.

According to Table 10, the best performance in terms of SSIM is obtained by using MFKM codebooks-the highest SSIM values are observed for MFKM in five out of seven training sets. P-M-T is a training set corresponding to the concatenation of images Peppers, Mandrill and Tiffany. It is 
important to point out that, for a fixed training set (with the exception of Lena), the absolute difference between the best SSIM result and the worst SSIM result is below 0.0090 .

Table 10. SSIM for images Lena, Barbara Elaine, Boat, Clock, Goldhill and P-M-T, using N = 32 .

\begin{tabular}{cccccccc}
\hline \multirow{2}{*}{ Algorithm } & \multicolumn{7}{c}{ SSIM } \\
\cline { 2 - 7 } & Lena & Barbara & Elaine & Boat & Clock & Goldhill & P-M-T \\
\hline KM & 0.7790 & 0.6800 & 0.7637 & 0.7081 & 0.8373 & 0.7078 & 0.7492 \\
FKM & 0.7838 & 0.6809 & 0.7687 & 0.7118 & 0.8447 & 0.7105 & 0.7501 \\
MFKM & 0.7840 & 0.6807 & 0.7688 & 0.7120 & 0.8457 & 0.7111 & 0.7496 \\
FKM1 & 0.7816 & 0.6807 & 0.7678 & 0.7109 & 0.8381 & 0.7105 & 0.7481 \\
MFKM1 & 0.7813 & 0.6813 & 0.7674 & 0.7095 & 0.8386 & 0.7110 & 0.7502 \\
FKM1-PDS & 0.7816 & 0.6807 & 0.7678 & 0.7109 & 0.8381 & 0.7105 & 0.7481 \\
MFKM1-PDS & 0.7813 & 0.6813 & 0.7674 & 0.7095 & 0.8386 & 0.7110 & 0.7502 \\
FKM1-ENNS & 0.7816 & 0.6807 & 0.7678 & 0.7109 & 0.8381 & 0.7105 & 0.7481 \\
MFKM1-ENNS & 0.7813 & 0.6813 & 0.7674 & 0.7095 & 0.8386 & 0.7110 & 0.7502 \\
FKM2 & 0.7731 & 0.6787 & 0.7617 & 0.7083 & 0.8383 & 0.7083 & 0.7483 \\
MFKM2 & 0.7736 & 0.6793 & 0.7622 & 0.7075 & 0.8395 & 0.7087 & 0.7490 \\
FKM2-PDS & 0.7731 & 0.6787 & 0.7617 & 0.7083 & 0.8383 & 0.7083 & 0.7483 \\
MFKM2-PDS & 0.7736 & 0.6793 & 0.7622 & 0.7075 & 0.8395 & 0.7087 & 0.7490 \\
FKM2-ENNS & 0.7731 & 0.6787 & 0.7617 & 0.7083 & 0.8383 & 0.7083 & 0.7483 \\
MFKM2-ENNS & 0.7736 & 0.6793 & 0.7622 & 0.7075 & 0.8395 & 0.7087 & 0.7490 \\
\hline
\end{tabular}

It is observed in Table 11 that MFKM leads to the highest SSIM values for five out of seven training sets. For a fixed training set (with the exception of Elaine and Clock), the absolute difference between the best SSIM result and the worst SSIM result is below 0.0090 .

Table 11. SSIM for images Lena, Barbara Elaine, Boat, Clock, Goldhill and P-M-T, using N = 64 .

\begin{tabular}{cccccccc}
\hline \multirow{2}{*}{ Algorithm } & \multicolumn{7}{c}{ SSIM } \\
\cline { 2 - 7 } & Lena & Barbara & Elaine & Boat & Clock & Goldhill & P-M-T \\
\hline KM & 0.8225 & 0.7323 & 0.8094 & 0.7652 & 0.8667 & 0.7613 & 0.7897 \\
FKM & 0.8260 & 0.7325 & 0.8136 & 0.7657 & 0.8749 & 0.7628 & 0.7902 \\
MFKM & 0.8261 & 0.7318 & 0.8137 & 0.7653 & 0.8756 & 0.7631 & 0.7910 \\
FKM1 & 0.8228 & 0.7355 & 0.8105 & 0.7655 & 0.8656 & 0.7629 & 0.7900 \\
MFKM1 & 0.8224 & 0.7351 & 0.8096 & 0.7643 & 0.8661 & 0.7622 & 0.7902 \\
FKM1-PDS & 0.8228 & 0.7355 & 0.8105 & 0.7655 & 0.8657 & 0.7629 & 0.7900 \\
MFKM1-PDS & 0.8224 & 0.7351 & 0.8096 & 0.7643 & 0.8661 & 0.7622 & 0.7902 \\
FKM1-ENNS & 0.8228 & 0.7355 & 0.8105 & 0.7655 & 0.8656 & 0.7629 & 0.7900 \\
MFKM1-ENNS & 0.8224 & 0.7351 & 0.8096 & 0.7643 & 0.8661 & 0.7622 & 0.7902 \\
FKM2 & 0.8177 & 0.7312 & 0.8031 & 0.7646 & 0.8680 & 0.7605 & 0.7900 \\
MFKM2 & 0.8179 & 0.7316 & 0.8032 & 0.7645 & 0.8692 & 0.7610 & 0.7897 \\
FKM2-PDS & 0.8177 & 0.7312 & 0.8031 & 0.7646 & 0.8680 & 0.7604 & 0.7900 \\
MFKM2-PDS & 0.8179 & 0.7316 & 0.8032 & 0.7645 & 0.8692 & 0.7610 & 0.7897 \\
FKM2-ENNS & 0.8177 & 0.7312 & 0.8031 & 0.7646 & 0.8680 & 0.7605 & 0.7900 \\
MFKM2-ENNS & 0.8179 & 0.7316 & 0.8032 & 0.7645 & 0.8692 & 0.7610 & 0.7897 \\
\hline
\end{tabular}

For $N=256$, it is observed in Table 13 that MFKM leads to the best SSIM results for 5 out of 7 traning sets considered. An interesting performance nuance must be pointed out-MFKM2, MFKM2-PDS and MFKM2-ENNS are the techniques that lead to the highest PSNR results (according to Tables 2-9), but do not lead to the best SSIM results (as can be observed from Tables 10-13). It is important to observe that codebook design aims to decrease the distortion (mean square error) obtained in representing the training vectors by the corresponding nearest neighbors, that is, by the corresponding codevectors with minimum distance. In other words, higher PSNR values are obtained by codebooks that are more "tuned" with the training set, that is, by codebooks that introduce less distortion in terms of MSE, which do not necessarily correspond to higher SSIM values. PSNR and SSIM results are presented in Table 14 for images reconstructed by codebooks designed with the training set P-M-T. 
The method MFKM2-ENNS was used for codebooks designed for $K=16$ and $N=32,64,128$ and 256 , leading to corresponding code rates $0.3125 \mathrm{bpp}, 0.375 \mathrm{bpp}, 0.4375 \mathrm{bpp}$ and $0.5 \mathrm{bpp}$. It is observed that, for a given image, both PSNR and SSIM increases with $N$, that is, the distortion decreases with the code rate.

Table 12. SSIM for images Lena, Barbara Elaine, Boat, Clock, Goldhill and P-M-T, using $N=128$.

\begin{tabular}{cccccccc}
\hline \multirow{2}{*}{ Algorithm } & \multicolumn{7}{c}{ SSIM } \\
\cline { 2 - 7 } & Lena & Barbara & Elaine & Boat & Clock & Goldhill & P-M-T \\
\hline KM & 0.8583 & 0.7863 & 0.8487 & 0.8141 & 0.8941 & 0.8050 & 0.8232 \\
FKM & 0.8617 & 0.7864 & 0.8523 & 0.8143 & 0.9006 & 0.8066 & 0.8219 \\
MFKM & 0.8616 & 0.7864 & 0.8523 & 0.8138 & 0.9014 & 0.8063 & 0.8224 \\
FKM1 & 0.8579 & 0.7881 & 0.8481 & 0.8035 & 0.8876 & 0.8076 & 0.8231 \\
MFKM1 & 0.8575 & 0.7855 & 0.8475 & 0.8024 & 0.8889 & 0.8062 & 0.8233 \\
FKM1-PDS & 0.8579 & 0.7881 & 0.8481 & 0.8035 & 0.8876 & 0.8076 & 0.8231 \\
MFKM1-PDS & 0.8575 & 0.7856 & 0.8475 & 0.8024 & 0.8889 & 0.8062 & 0.8233 \\
FKM1-ENNS & 0.8579 & 0.7881 & 0.8481 & 0.8035 & 0.8876 & 0.8076 & 0.8231 \\
MFKM1-ENNS & 0.8575 & 0.7855 & 0.8475 & 0.8024 & 0.8889 & 0.8062 & 0.8233 \\
FKM2 & 0.8518 & 0.7823 & 0.8387 & 0.8124 & 0.8899 & 0.8041 & 0.8236 \\
MFKM2 & 0.8520 & 0.7833 & 0.8394 & 0.8128 & 0.8906 & 0.8048 & 0.8244 \\
FKM2-PDS & 0.8518 & 0.7823 & 0.8387 & 0.8124 & 0.8899 & 0.8041 & 0.8236 \\
MFKM2-PDS & 0.8520 & 0.7833 & 0.8394 & 0.8128 & 0.8906 & 0.8048 & 0.8244 \\
FKM2-ENNS & 0.8518 & 0.7823 & 0.8387 & 0.8124 & 0.8899 & 0.8041 & 0.8236 \\
MFKM2-ENNS & 0.8520 & 0.7833 & 0.8394 & 0.8128 & 0.8906 & 0.8048 & 0.8244 \\
\hline
\end{tabular}

Table 13. SSIM for images Lena, Barbara Elaine, Boat, Clock, Goldhill and P-M-T, using N = 256.

\begin{tabular}{cccccccc}
\hline \multirow{2}{*}{ Algorithm } & \multicolumn{7}{c}{ SSIM } \\
\cline { 2 - 7 } & Lena & Barbara & Elaine & Boat & Clock & Goldhill & P-M-T \\
\hline KM & 0.8893 & 0.8351 & 0.8808 & 0.8514 & 0.9173 & 0.8450 & 0.8534 \\
FKM & 0.8935 & 0.8371 & 0.8843 & 0.8540 & 0.9226 & 0.8478 & 0.8516 \\
MFKM & 0.8935 & 0.8372 & 0.8843 & 0.8539 & 0.9231 & 0.8479 & 0.8518 \\
FKM1 & 0.8875 & 0.8349 & 0.8764 & 0.8478 & 0.9095 & 0.8452 & 0.8530 \\
MFKM1 & 0.8877 & 0.8322 & 0.8761 & 0.8490 & 0.9100 & 0.8442 & 0.8529 \\
FKM1-PDS & 0.8875 & 0.8349 & 0.8764 & 0.8478 & 0.9095 & 0.8452 & 0.8530 \\
MFKM1-PDS & 0.8877 & 0.8322 & 0.8761 & 0.8490 & 0.9100 & 0.8442 & 0.8529 \\
FKM1-ENNS & 0.8875 & 0.8349 & 0.8764 & 0.8478 & 0.9096 & 0.8452 & 0.8530 \\
MFKM1-ENNS & 0.8877 & 0.8322 & 0.8761 & 0.8490 & 0.9100 & 0.8442 & 0.8529 \\
FKM2 & 0.8842 & 0.8333 & 0.8690 & 0.8520 & 0.9145 & 0.8450 & 0.8550 \\
MFKM2 & 0.8852 & 0.8339 & 0.8696 & 0.8527 & 0.9155 & 0.8460 & 0.8553 \\
FKM2-PDS & 0.8842 & 0.8333 & 0.8690 & 0.8520 & 0.9145 & 0.8450 & 0.8550 \\
MFKM2-PDS & 0.8852 & 0.8339 & 0.8696 & 0.8527 & 0.9155 & 0.8460 & 0.8553 \\
FKM2-ENNS & 0.8842 & 0.8333 & 0.8690 & 0.8519 & 0.9145 & 0.8450 & 0.8550 \\
MFKM2-ENNS & 0.8852 & 0.8339 & 0.8696 & 0.8527 & 0.9155 & 0.8460 & 0.8553 \\
\hline
\end{tabular}

Table 14. PSNR (in $\mathrm{dB}$ ) and SSIM of reconstructed images by using codebooks designed with the training set $\mathrm{P}-\mathrm{M}-\mathrm{T}$, using MFKM2-ENNS.

\begin{tabular}{ccccccccc}
\hline \multirow{2}{*}{ Images } & \multicolumn{2}{c}{$N=\mathbf{3 2}$} & \multicolumn{2}{c}{$N=\mathbf{6 4}$} & \multicolumn{2}{c}{$N=\mathbf{1 2 8}$} & \multicolumn{2}{c}{$N=\mathbf{2 5 6}$} \\
\cline { 2 - 9 } & PSNR & SSIM & PSNR & SSIM & PSNR & SSIM & PSNR & SSIM \\
\hline Lena & 25.62 & 0.7211 & 26.34 & 0.7604 & 26.91 & 0.7816 & 27.50 & 0.8133 \\
Barbara & 24.09 & 0.6350 & 24.66 & 0.6679 & 25.19 & 0.6982 & 25.68 & 0.7293 \\
Elaine & 26.62 & 0.7223 & 27.51 & 0.7626 & 28.11 & 0.7848 & 28.88 & 0.8134 \\
Boat & 24.16 & 0.6575 & 24.88 & 0.7038 & 25.31 & 0.7259 & 25.89 & 0.7633 \\
Clock & 25.21 & 0.7991 & 26.05 & 0.8207 & 26.81 & 0.8470 & 27.32 & 0.8618 \\
Goldhill & 25.71 & 0.6391 & 26.34 & 0.6788 & 26.92 & 0.7132 & 27.45 & 0.7435 \\
Tiffany & 28.21 & 0.7493 & 29.10 & 0.7917 & 30.40 & 0.8365 & 31.38 & 0.8647 \\
\hline
\end{tabular}


The last set of simulations show that vector quantization in the Discrete Wavelet Transform (DWT) domain (that is, by quantizing the wavelet coefficients) lead to reconstructed images with better quality when compared to the ones obtained by VQ in the spatial domain (that is, by quantizing the gray scale values of pixels). For the purpose of DWT VQ [52] at the code rate $0.3125 \mathrm{bpp}$, a three level multiresolution wavelet decomposition was performed [53] with the wavelet family Daubechies 6. The resulting subbands $S_{i j}$ are submitted to quantization schemes according to Figure 2.

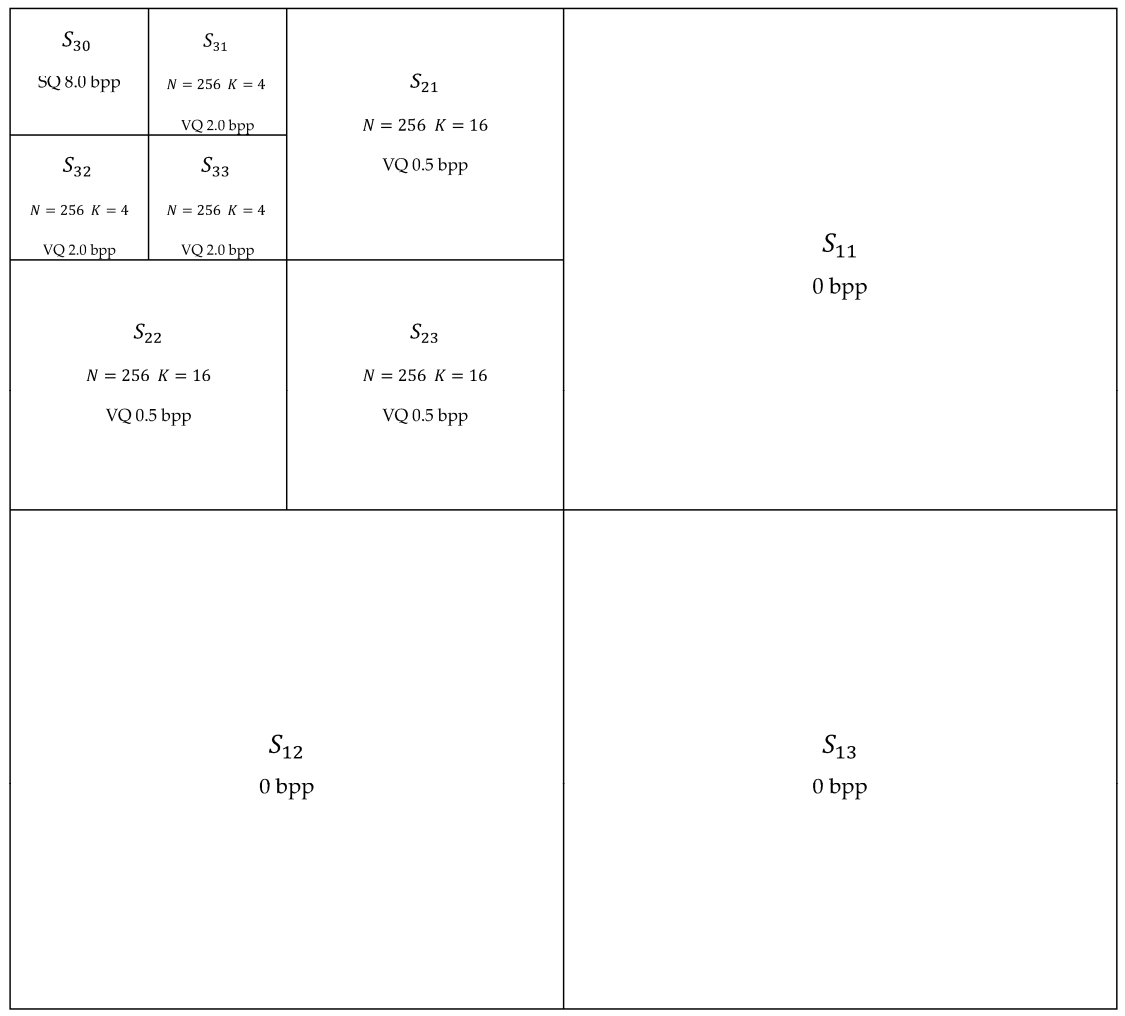

Figure 2. Image encoding using DWT.

Subbands $S_{21}, S_{22}$ and $S_{23}$ are submitted to the respective wavelet VQ codebooks with $N=256$ and $K=16$ (blocks of $4 \times 4$ wavelet coefficents). Subbands $S_{31}, S_{32}$ and $S_{33}$ are submitted to the respective wavelet VQ codebooks with $N=256$ and $K=4$ (blocks of $2 \times 2$ wavelet coefficents). Subband $S_{30}$ is submitted to scalar quantization (SQ) with $8.0 \mathrm{bpp}$. Subbands $S_{11}, S_{12}$ and $S_{13}$ are excluded (that is, code rate $0 \mathrm{bpp}$ ) —one can observe in Figure 3 that the application of the inverse discrete wavelet transform after exclusion of subbands $S_{11}, S_{12}$ and $S_{13}$, preserving all the other subbands with the wavelet coefficients unchanged, leads to images close to the respective original ones (Figure 1), with good quality, as revealed by visual inspection.

It is worth mentioning that, in the general case, after the application of a multiresolution discrete wavelet transform (DWT) with $L$ resolution levels, the subbands $S_{i j}$, with $i=1,2 \ldots, L$ and $j=1,2,3$, are submitted to multiresolution VQ codebooks. In other words, with the exception of subband $S_{L 0}$ (corresponding to the approximation component in the lowest resolution level), each subband is quantized with a specific codebook. The subband $S_{L 0}$ is submitted to 8.0 bpp scalar quantization, since it is the subband with the highest importance to the quality of the image obtained from the inverse discrete wavelet transform (IDWT).

Assume the general case of an image with $P \times P$ pixels. The number of wavelet coefficients in $S_{i j}$, with $1 \leq i \leq L$, is $\frac{P \times P}{2^{i} \times 2^{i}}$. Let $R_{S_{i j}}$ be the code rate (in bpp or, correspondingly, in bit/coefficient) of VQ for subband $S_{i j}, 1 \leq i \leq L$ and $1 \leq j \leq 3$, and $R_{S_{L 0}}$ be the code rate (in bpp) of scalar quantization for subband $S_{L 0}$. The final code rate $R_{T}$ (in bpp) of the image coding using DWT (with $L$ resolution levels) and VQ is given by: 


$$
R_{T}=\frac{1}{P \times P}\left(\frac{P \times P}{2^{L} \times 2^{L}} R_{S_{L 0}}+\sum_{i=1}^{L} \sum_{j=1}^{3} \frac{P \times P}{2^{i} \times 2^{i}} R_{S_{i j}}\right),
$$

that is:

$$
R_{T}=\frac{R_{S_{L 0}}}{2^{2 L}}+\sum_{i=1}^{L} \sum_{j=1}^{3} \frac{R_{S_{i j}}}{2^{2 i}} .
$$

For VQ with dimension $K$ and codebook size $N$, it follows that the corresponding code rate is $\frac{1}{K} \log _{2} N$. Hence, according to Figure 2, it follows that:

$$
R_{S_{21}}=R_{S_{22}}=R_{S_{23}}=\frac{1}{16} \log _{2} 256=0.5 \mathrm{bpp}
$$

and:

$$
R_{S_{31}}=R_{S_{32}}=R_{S_{33}}=\frac{1}{4} \log _{2} 256=2.0 \mathrm{bpp}
$$

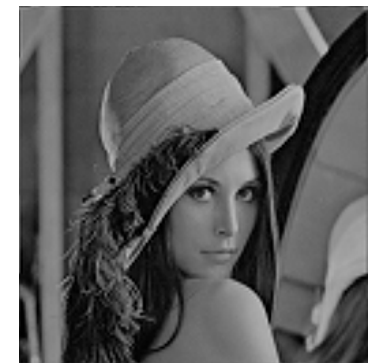

(a)

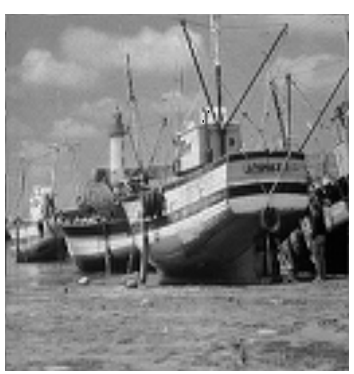

(d)

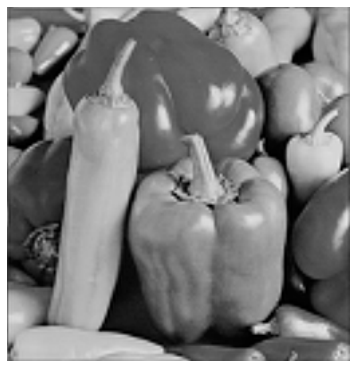

(g)

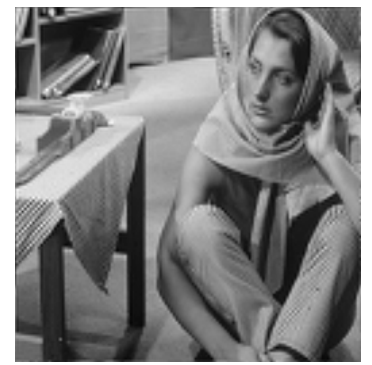

(b)

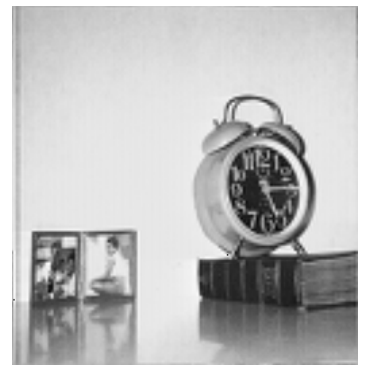

(e)

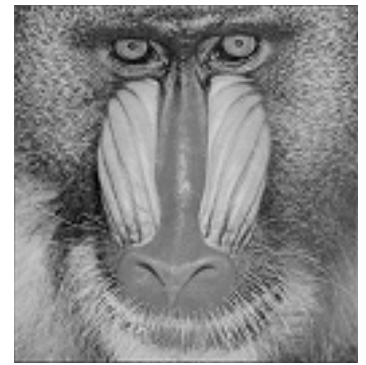

(h)

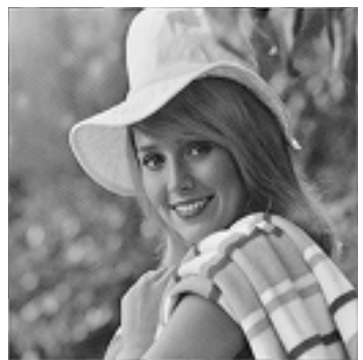

(c)

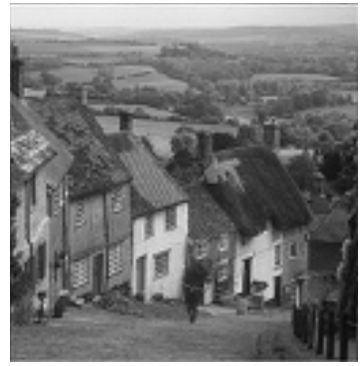

(f)

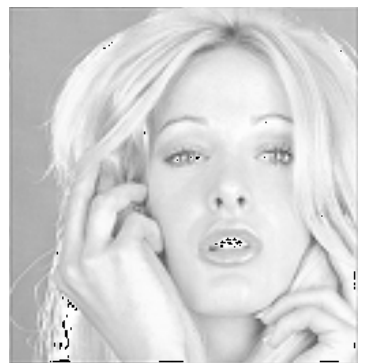

(i)

Figure 3. Images obtained from the inverse discrete wavelet transform with the exclusion of subbands $S_{11}, S_{12}$ and $S_{13}$. (a) Lena PSNR = $30.05 \mathrm{~dB}$; (b) Barbara PSNR = $25.54 \mathrm{~dB}$; (c) Elaine PSNR = $31.88 \mathrm{~dB}$; (d) Boat PSNR = 26.07 dB; (e) Clock PSNR = 29.02 dB; (f) Goldhill PSNR = 27.77 dB; (g) Peppers PSNR = 30.74 dB; (h) Mandrill PSNR = $24.93 \mathrm{~dB}$; (i) Tiffany PSNR = $31.69 \mathrm{~dB}$.

From Figure 2, it follows that $R_{S_{30}}=8.0 \mathrm{bpp}$ and $R_{S_{11}}=R_{S_{12}}=R_{S_{13}}=0 \mathrm{bpp}$. Thus, from Equation (17), the corresponding overall code rate under the conditions presented in Figure 2 is $R_{T}=0.3125 \mathrm{bpp}$. It is worth mentioning that the importance of subbands $S_{i j}$ for the image quality increases with $i$ that is the reason why $R_{S_{3 j}}>R_{S_{2 j}}$, for $j=1,2,3$. 
As can be observed in Figures 4 and 5, visual inspections of the reconstructed images reveal the superiority of DWT VQ over vector quantization in the spatial domain. The superiority is also confirmed in terms of PSNR and SSIM values.

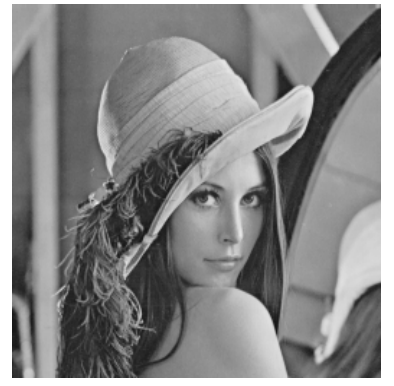

(a)

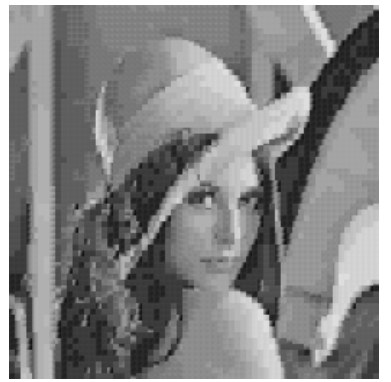

(b)

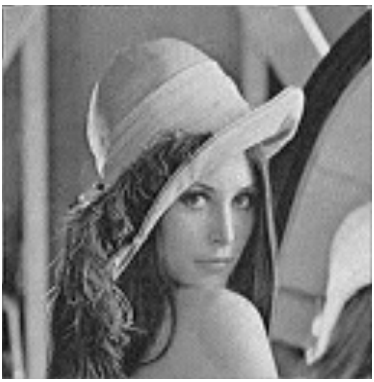

(c)

Figure 4. Images Lena: (a) Original; (b) Reconstructed using spatial domain VQ with 0.3125 bpp (PSNR = $25.62 \mathrm{~dB}$ and SSIM = 0.7211); (c) Reconstructed using DWT VQ with $0.3125 \mathrm{bpp}$ (PSNR = $29.35 \mathrm{~dB}$ and SSIM $=0.8367)$. Codebooks were designed with training set P-M-T by MFKM2-ENNS.

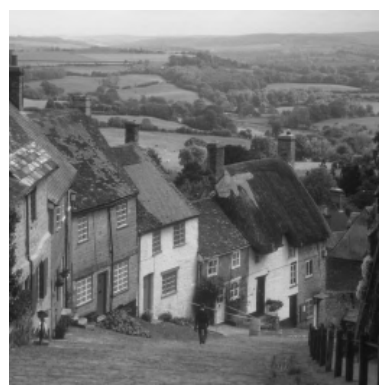

(a)

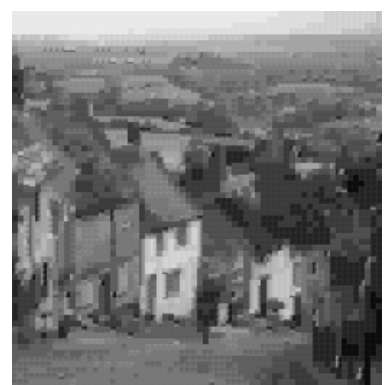

(b)

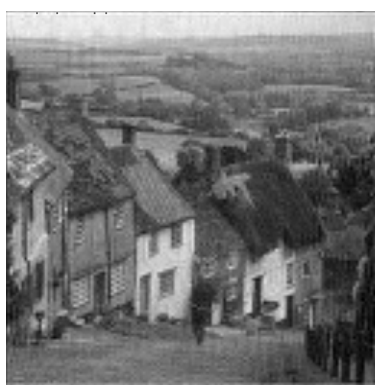

(c)

Figure 5. Images Goldhill: (a) Original; (b) Reconstructed using spatial domain VQ with 0.3125 bpp (PSNR = 25.71 dB and SSIM = 0.6391); (c) Reconstructed using DWT VQ with $0.3125 \mathrm{bpp}$ (PSNR = $26.81 \mathrm{~dB}$ and SSIM $=0.7640$ ). Codebooks were designed with training set P-M-T by MFKM2-ENNS.

The superiority of DWT VQ over spatial domain VQ is also observed in Table 15. As an example, by using P-M-T as the training set, PSNR gain of $3.10 \mathrm{~dB}$ for Elaine image is obtained by substituting spatial domain VQ by DWT VQ. For a given image, one can observe that better PSNR and SSIM results are obtained by DWT VQ with codebooks designed by P-M-T when compared to spatial domain VQ with codebook designed by the image itself. Consider, for instance, the Lena image. If the Lena image is reconstructed using spatial domain VQ with codebook designed by itself as training set, a PSNR $26.72 \mathrm{~dB}$ and a SSIM 0.7791 are obtained. If the Lena image is reconstructed in the DWT domain with multiresolution codebooks designed by P-M-T as training set, a PSNR $29.35 \mathrm{~dB}$ and a SSIM 0.8367 are obtained.

As a final comment, image coding based on VQ is one of the possible applications of the families of fuzzy K-means algorithms considered in this paper. The focus of the present work is to assess the fact that the proposed acceleration techniques make VQ codebook design faster, since other efficient image coding techniques exist. 
Table 15. PSNR (in $\mathrm{dB}$ ) and SSIM of reconstructed images. Codebooks were designed using MFKM2-ENNS in spatial domain as well as by the DWT domain for code rate $0.3125 \mathrm{bpp}$.

\begin{tabular}{ccccccc}
\hline Images & \multicolumn{2}{c}{$\begin{array}{c}\text { Spatial Domain VQ. } \\
\text { Performance Inside the } \\
\text { Training Set }\end{array}$} & \multicolumn{2}{c}{$\begin{array}{c}\text { Spatial Domain VQ with } \\
\text { Codebooks Designed by } \\
\text { Using P-M-T Training Set }\end{array}$} & $\begin{array}{c}\text { DWT VQ with Multiresolution } \\
\text { Codebooks Designed by Using } \\
\text { P-M-T Training Set }\end{array}$ \\
\cline { 2 - 7 } & PSNR & SSIM & PSNR & SSIM & PSNR & SSIM \\
\hline Lena & 26.72 & 0.7791 & 25.62 & 0.7211 & 29.35 & 0.8367 \\
Barbara & 24.78 & 0.6822 & 24.09 & 0.6350 & 25.00 & 0.7573 \\
Elaine & 27.79 & 0.7566 & 26.62 & 0.7223 & 29.72 & 0.8304 \\
Boat & 24.90 & 0.7047 & 24.16 & 0.6575 & 25.49 & 0.7581 \\
Clock & 26.27 & 0.8364 & 25.21 & 0.7991 & 28.22 & 0.8672 \\
Goldhill & 26.76 & 0.7085 & 25.71 & 0.6391 & 26.81 & 0.7640 \\
Tiffany & 29.01 & 0.8078 & 28.21 & 0.7493 & 30.21 & 0.8099 \\
\hline
\end{tabular}

\section{Conclusions}

In this work, alternatives were presented for accelerating families of fuzzy K-means algorithms applied to vector quantization codebook design. A lookahead approach was used with the purpose of decreasing the number of iterations of the algorithms. The approach consists in using a scale factor in the computation of the codevectors.

An additional acceleration was obtained by accommodating efficient nearest neighbor search techniques in the partitioning step of the algorithms. With such approach, savings are obtained in the number of operations spent by the algorithms. The combination of the scale factor (lookahead approach) with efficient nearest neighbor search was evaluated in the scenario of image vector quantization codebook design. Savings up to $40 \%$ in the time spent for codebook design were obtained, without sacrificing the quality of the codebook, assessed by the peak signal-to-noise ratio (PSNR) as well as by structural similarity (SSIM) index of the reconstructed images.

Acknowledgments: The authors would like to thank CNPq and PIBITI program of the Catholic University of Pernambuco for supporting this research. The authors also would like to thank the anonymous reviewers for their valuable comments and suggestions to improve the quality of the paper.

Author Contributions: Edson Mata and Francisco Madeiro have proposed the alternative of accelerating fuzzy K-means algorithms. Edson Mata implemented the techniques, with the support of Silvio Bandeira. All the authors have contributed to simulation results analysis and to the manuscript writing. All authors have read and approved the final manuscript.

Conflicts of Interest: The authors declare no conflict of interest.

\section{References}

1. Gersho, A.; Gray, R.M. Vector Quantization and Signal Compression; Kluwer Academic Publishers: Boston, MA, USA, 1992.

2. Gray, R.M. Vector Quantization. IEEE ASSP Mag. 1984, 1, 4-29. [CrossRef]

3. Ma, Z.; Taghia, J.; Kleijn, W.B.; Guo, J. Line Spectral Frequencies Modeling by a Mixture of Von Mises-Fisher Distributions. Signal Proc. 2015, 114, 219-224. [CrossRef]

4. Paliwal, K.K.; Atal, B.S. Efficient Vector Quantization of LPC Parameters at 24 Bits/Frame. IEEE Trans. Audio Speech Lang. Proc. 1993, 1, 3-14. [CrossRef]

5. Yahampath, P.; Rondeau, P. Multiple-Description Predictive-Vector Quantization with Applications to Low Bit-Rate Speech Coding Over Networks. IEEE Trans. Audio Speech Lang. Proc. 2007, 15, 749-755. [CrossRef]

6. Akhtarkavan, E.; Salleh, M.F.M. Multiple Descriptions Coinciding Lattice Vector Quantizer for Wavelet Image Coding. IEEE Trans. Image Proc. 2012, 21, 653-661. [CrossRef] [PubMed]

7. Tsolakis, D.; Tsekouras, G.E.; Tsimikas, J. Fuzzy Vector Quantization for Image Compression Based on Competitive Agglomeration and a Novel Codeword Migration Strategy. Eng. Appl. Artif. Intell. 2012, 25, 1212-1225. [CrossRef] 
8. Wen, J.; Ma, C.; Zhao, J. FIVQ Algorithm for Interference Hyper-Spectral Image Compression. Opt. Commun. 2014, 322, 97-104. [CrossRef]

9. Hu, Y.C.; Chen, W.L.; Lo, C.C.; Wu, C.M.; Wen, C.H. Efficient VQ-Based Image Coding Scheme Using Inverse Function and Lossless Index Coding. Signal Proc. 2013, 93, 2432-2439. [CrossRef]

10. Hanilçi, C.; Ertas, F. Investigation of the Effect of Data Duration and Speaker Gender on Text-Independent Speaker Recognition. Comput. Electr. Eng. 2013, 39, 441-452. [CrossRef]

11. Madeiro, F.; Fechine, J.M.; Lopes, W.T.A.; Aguiar Neto, B.G.; Alencar, M.S. Identificação Vocal por Frequência Fundamental, QV e HMMS. In Em-TOM-Ação: A Prosódia em Perspectiva, 1st ed.; Aguiar, M.A.M., Madeiro, F., Eds.; Editora Universitária da UFPE: Recife, Brazil, 2007; pp. 91-120.

12. Qin, C.; Chang, C.-C. A Novel Joint Data-Hiding and Compression Scheme Based on SMVQ and Image Inpainting. IEEE Trans. Image Proc. 2014, 23, 969-978.

13. Chang, C.-C.; Wu, W.-C. Hiding Secret Data Adaptively in Vector Quantisation Index Tables. IEEE Proc. Vis. Image Signal Proc. 2006, 153, 589-597. [CrossRef]

14. Qin, C.; Hu, Y.-C. Reversible Data Hiding in VQ Index Table with Lossless Coding and Adaptive Switching Mechanism. Signal Proc. 2016, 129, 48-55. [CrossRef]

15. Chang, C.C.; Nguyen, T.S.; Lin, C.C. A Reversible Compression Code Hiding Using SOC and SMVQ Indices. Inf. Sci. 2015, 300, 85-99. [CrossRef]

16. Tu, T.Y.; Wang, C.H. Reversible Data Hiding with High Payload Based on Referred Frequency for VQ Compressed Codes Index. Signal Proc. 2015, 108, 278-287. [CrossRef]

17. Kieu, T.D.; Ramroach, S. A Reversible Steganographic Scheme for VQ Indices Based on Joint Neighboring Coding. Exp. Syst. Appl. 2015, 42, 713-722. [CrossRef]

18. Hu, H.T.; Hsu, L.Y.; Chou, H.H. Variable-Dimensional Vector Modulation for Perceptual-Based DWT Blind Audio Watermarking with Adjustable Payload Capacity. Digit. Signal Proc. 2014, 31, 115-123. [CrossRef]

19. Vieira, R.T.; Brunet, N.; Costa, S.C.; Correia, S.; Aguiar Neto, B.G.; Fechine, J.M. Combining Entropy Measurements and Cepstral Analysis for Pathological Voice Assessment. J. Med. Biol. Eng. 2012, 32, 429-435. [CrossRef]

20. Linde, Y.; Buzo, A.; Gray, R.M. An Algorithm for Vector Quantizer Design. IEEE Trans. Commun. 1950, 28, 84-95. [CrossRef]

21. Tsolakis, D.; Tsekouras, G.E.; Niros, A.D.; Rigos, A. On the Systematic Development of Fast Fuzzy Vector Quantization for Grayscale Image Compression. Inf. Sci. 2012, 36, 83-96. [CrossRef] [PubMed]

22. Hunga, W.-L.; Chen, D.-H.; Yang, M.-S. Suppressed Fuzzy-Soft Learning Vector Quantization for MRI Segmentation. Inf. Sci. 2011, 52, 33-43. [CrossRef] [PubMed]

23. Krishnamurthy, A.K.; Ahalt, S.C.; Melton, D.E.; Chen, P. Neural Networks for Vector Quantization of Speech and Images. IEEE J. Sel. Areas Commun. 1990, 8, 1449-1457. [CrossRef]

24. Azevedo, C.R.B.; Azevedo, F.E.A.G.; Lopes, W.T.A.; Madeiro, F. Terrain-Based Memetic Algorithms to Vector Quantization Design. In Nature Inspired Cooperative Strategies for Optimization (NICSO 2008); Krasnogor, N., Ed.; DEU: Berlin, Germany, 2009; Volume 236, pp. 197-211.

25. Pan, J.S.; Mclnnes, F.R.; Jack, M.A. VQ Codebook Design Using Genetic Algorithms. Electron. Lett. 1995, 31, 1418-1419. [CrossRef]

26. Horng, M.-H. Vector Quantization Using the Firefly Algorithm for Image Compression. Inf. Sci. 2012, 39, 1078-1091. [CrossRef]

27. Horng, M.-H.; Jiang, T.-W. Image Vector Quantization Algorithm via Honey Bee Mating Optimization. Inf. Sci. 2012, 38, 1382-1392. [CrossRef]

28. Karayiannis, N.B.; Bezdek, J.C. An Integrated Approach to Fuzzy Learning Vector Quantization and Fuzzy C-Means Clustering. IEEE Trans. Fuzzy Syst. 1997, 5, 622-628. [CrossRef]

29. Karayiannis, N.B.; Pai, P.I. Fuzzy Vector Quantization Algorithms and Their Application in Image Compression. IEEE Trans. Image Proc. 1995, 4, 1193-1201. [CrossRef] [PubMed]

30. Tsao, E.C.K.; Bezdek, J.C.; Pal, N.R. Fuzzy Kohonen Clustering Networks. Pattern Recognit. 1994, $27,757-764$. [CrossRef]

31. Tsekouras, G.E.; Mamalis, A.; Anagnostopoulos, C.; Gavalas, D.; Economou, D. Improved Batch Fuzzy Learning Vector Quantization for Image Compression. Inf. Sci. 2008, 178, 3895-3907. [CrossRef]

32. Triantafilisa, J.; Gibbsb, I.; Earla, N. Digital Soil Pattern Recognition in the Lower Namoi Valley Using Numerical Clustering of Gamma-Ray Spectrometry Data. Geoderma 2013, 192, 407-421. [CrossRef] 
33. Malinverni, E.S.; Fangi, G. Comparative Cluster Analysis to Localize Emergencies in Archaeology. J. Cult. Herit. 2009, 10, e10-e19. [CrossRef]

34. Chen, G.; Meng, X.; Wang, Y.; Zhang, Y.; Tian, P.; Yang, H. Integrated WiFi/PDR/Smartphone Using an Unscented Kalman Filter Algorithm for 3D Indoor Localization. Sensors 2015, 15, 24595-24614. [CrossRef] [PubMed]

35. Fernández, R.; Montes, H.; Salinas, C.; Sarria, J.; Armada, M. Combination of RGB and Multispectral Imagery for Discrimination of Cabernet Sauvignon Grapevine Elements. Sensors 2013, 13, 7838-7859. [CrossRef] [PubMed]

36. Zhang, C.; Xiao, X.; Li, X.; Chen, Y.-J.; Zhen, W.; Chang, J.; Zheng, C.; Liu, Z. White Blood Cell Segmentation by Color-Space-Based K-Means Clustering. Sensors 2014, 14, 16128-16147. [CrossRef] [PubMed]

37. Chen, C.-H.; Huang, W.-T.; Tan, T.-H.; Chang, C.-C.; Chang, Y.-J. Using K-Nearest Neighbor Classification to Diagnose Abnormal Lung Sounds. Sensors 2015, 15, 13132-13158. [CrossRef] [PubMed]

38. Liu, N.; Cao, W.; Zhu, Y.; Zhang, J.; Pang, F.; Ni, J. The Node Deployment of Intelligent Sensor Networks Based on the Spatial Difference of Farmland Soil. Sensors 2015, 15, 28314-28339. [CrossRef] [PubMed]

39. Adhikaria, S.K.; Singb, J.K.; Basub, D.K.; Nasipurib, M. Conditional Spatial Fuzzy C-Means Clustering Algorithm for Segmentation of MRI Images. Appl. Soft Comput. 2015, 34, 758-769. [CrossRef]

40. Mekhmoukh, A.; Mokrani, K. Improved Fuzzy C-Means Based Particle Swarm Optimization (PSO) Initialization and Outlier Rejection with Level Set Methods for MR Brain Image Segmentation. Comput. Methods Prog. Biomed. 2015, 122, 266-281. [CrossRef] [PubMed]

41. Kinnunen, T.; Sidoroff, I.; Tuononen, M.; Fränti, P. Comparison of Clustering Methods: A Case Study of Text-Independent Speaker Modeling. Pattern Recognit. Lett. 2011, 32, 1604-1617. [CrossRef]

42. Alkhalaf, S.; Alfarraj, O.; Hemeida, A.M. Fuzzy-VQ Image Compression based Hybrid PSOGSA Optimization Algorithm. In Proceedings of the IEEE International Conference on Fuzzy Systems (FUZZ-IEEE), Istanbul, Turkey, 2-5 August 2015; pp. 1-6.

43. Bhattacharyya, P.; Mitra, A.; Chatterjee, A. Vector Quantization Based Image Compression Using Generalized Improved Fuzzy Clustering. In Proceedings of the International Conference on Control, Instrumentation, Energy and Communication (CIEC14), Kolkata, India, 31 January-2 February 2014; pp. 662-666.

44. Guan, L.; Kamel, M. Equal-Average Hyperplane Partitioning Method for Vector Quantization of Image Data. Pattern Recognit. Lett. 1992, 13, 693-699. [CrossRef]

45. Ra, S.W.; Kim, J.K. A Fast Mean-Distance-Ordered Partial Codebook Search Algorithm for Image Vector Quantization. IEEE Trans. Circuits Syst. II 1993, 40, 576-579. [CrossRef]

46. Lee, D.; Baek, S.; Sung, K. Modified K-Means Algorithm for Vector Quantizer Design. IEEE Signal Proc. Lett. 1997, 4, 2-4.

47. Paliwal, K.K.; Ramasubramanian, V. Comments on Modified K-Means Algorithm for Vector Quantizer Design. IEEE Trans. Image Proc. 2000, 9, 1964-1967. [CrossRef] [PubMed]

48. Bei, C.D.; Gray, R.M. An Improvement of the Minimum Distortion Enconding Algorithm for Vector Quantization. IEEE Trans. Commun. 1985, 33, 1132-1133.

49. Chu, S.C.; Lu, Z.M.; Pan, J.S. Hadamard Transform Based Fast Codeword Search Algorithm for High-Dimensional VQ Enconding. Inf. Sci. 2007, 177, 734-746. [CrossRef]

50. Chang, C.-C.; Wu, W.-C. Fast Planar-Oriented Ripple Search Algorithm for Hyperspace VQ Codebook. IEEE Trans. Image Proc. 2007, 16, 1538-1547. [CrossRef]

51. Wang, Z.; Bovik, A.C.; Sheikh, H.R. Image Quality Assessment: From Error Visibility to Structural Similarity. IEEE Trans. Image Proc. 2004, 13, 600-612. [CrossRef]

52. Averbuch, A.; Lazar, D.; Israeli, M. Image Compression Using Wavelet Transform and Multiresolution Decomposition. IEEE Trans. Image Proc. 1996, 5, 4-15. [CrossRef] [PubMed]

53. Mallat, S.G. A Theory for Multiresolution Signal Decomposition: The Wavelet Representation. IEEE Trans. Pattern Anal. Mach. Int. 1996, 5, 4-15.

(C) 2016 by the authors; licensee MDPI, Basel, Switzerland. This article is an open access article distributed under the terms and conditions of the Creative Commons Attribution (CC-BY) license (http://creativecommons.org/licenses/by/4.0/). 